Supporting Information

\title{
A Convergent Synthesis of the Macrocyclic Core of Cytotrienins: Application of RCM for Macrocyclization
}

\author{
Gwilherm Evano, Jennifer V. Schaus and James S. Panek* \\ Department of Chemistry and Center for Chemical Methodology and \\ Library Development, Boston University, 590 Commonwealth Avenue, \\ Boston, Massachusetts 02215.
}

General Information. All reactions were carried out in oven or flame-dried glassware under an argon atmosphere employing standard techniques in handling air-sensitive materials. All solvents were reagent grade. Tetrahydrofuran (THF) and diethyl ether $\left(\mathrm{Et}_{2} \mathrm{O}\right)$ were freshly distilled from sodium/benzophenone under argon immediately prior to use. Dichloromethane, benzene, toluene and acetonitrile were freshly distilled from calcium hydride. Methanol was distilled over magnesium/iodine. Triethylamine, diisopropylethylamine, pyridine, $N, N^{\prime}$ dimethylethylenediamine and 2,6-lutidine were distilled over calcium hydride and stored over potassium hydroxide. $N, N$-Dimethylformamide and dimethylsulfoxide were distilled over calcium hydride and stored over $4 \AA$ molecular sieves. $n$-Butyllithium was purchased from Aldrich and standardized by titration with menthol/1,10-phenanthroline. Oxalyl chloride was freshly distilled from quinoline before each use. Boron trifluoride diethyletherate was distilled immediately before use. Copper(I) iodide (99,999 \% purity) and Grubbs $1^{\text {st }}$ generation catalyst were purchased from Aldrich and used as supplied. Grubbs $2^{\text {nd }}$ generation catalyst was purchased 
from Strem Chemicals and used as supplied. Lithium bis(trimethylsilyl)amide solution was freshly prepared from 1,1,1,3,3,3-hexamethyldisilylazane and n-butyllithium before use. All other reagents were used as supplied. Unless otherwise noted, reactions were magnetically stirred and monitored by thin layer chromatography with Sorbent Technologies $0.20 \mathrm{~mm}$ silica gel 60 plates. Flash chromatography were performed with silica gel 60 (particle size 0.032-0.063 mm) supplied by Sorbent Technologies. Reverse phase chromatography was performed with Waters Sep-Pak ${ }^{\circledR}$ Vac 12cc (2g) C18 cartridges. For reactions run on a scale smaller than $20 \mathrm{mg}$, solvents used for extraction and purifications were distilled before use. Yields refer to chromatographically and spectroscopically pure compounds, unless otherwise noted. Proton NMR spectra were recorded using an internal deuterium lock at ambient temperature on a Varian $400 \mathrm{MHz}$ spectrometer. An internal reference of $\delta_{\mathrm{H}} 7.26$ was used for $\mathrm{CDCl}_{3}$. Data are presented as follows: chemical shift (in ppm on the $\delta$ scale relative to $\delta_{\mathrm{TMS}}=0$ ), multiplicity ( $\mathrm{s}=$ singlet, $\mathrm{d}$ = doublet, $\mathrm{t}=$ triplet, $\mathrm{q}=$ quartet, quint. = quintuplet, $\mathrm{m}=$ multiplet, $\mathrm{br}=$ broad, $\mathrm{ABq}=\mathrm{AB}$ quartet), coupling constant $(\mathrm{J} / \mathrm{Hz})$ and integration. Resonances that are either partially or fully obscured are denoted obscured (obsc). Carbon-13 NMR spectra were recorded on a Varian 75 $\mathrm{MHz}$ spectrometer. An internal reference of $\delta_{\mathrm{C}} 77.16$ was used for $\mathrm{CDCl}_{3}$. Infrared spectra were recorded on a Nexus 670 FTIR spectrophotometer. Optical rotations were recorded on an Autopol III digital polarimeter at $589 \mathrm{~nm}$ and reported as follows: $[\alpha]_{\mathrm{D}}^{20}$, concentration $(c$ in g/100 mL) and solvent. High-resolution mass-spectra were obtained on a Finnagan MAT-90 spectrometer in the Boston University Mass Spectrometry Laboratory. 


\section{Experimental Procedures}

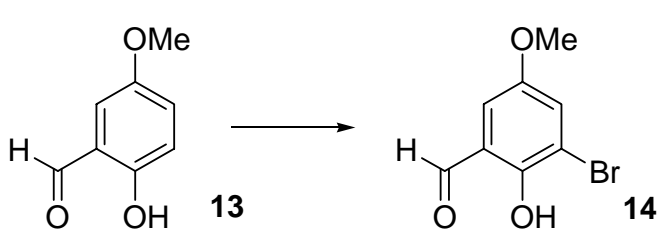

3-Bromo-2-hydroxy-5-methoxy-benzaldehyde 14. To a solution of 2-hydroxy-5methoxybenzaldehyde (10.0 g, $66 \mathrm{mmol})$ in glacial acetic acid (300 mL) was added sodium acetate $(8.6 \mathrm{~g}, 105 \mathrm{mmol})$ and bromine $(4.35 \mathrm{~mL}, 85 \mathrm{mmol})$. The resulting yellow mixture was stirred at rt for 1 hour and concentrated. The residue was then dissolved in $\mathrm{CH}_{2} \mathrm{Cl}_{2}(500 \mathrm{~mL})$, washed twice with $200 \mathrm{~mL}$ of water, dried over $\mathrm{MgSO}_{4}$, filtered and concentrated under vacuum. The crude solid was next recrystallized from ethanol to yield a yellow solid (13.6 g, 58.9 mmol, 89 \%). Mp : $108{ }^{\circ} \mathrm{C} ;{ }^{1} \mathrm{H}$ NMR (400 MHz, $\mathrm{CDCl}_{3}$ ): $\delta 11.11$ (s, 1H), 9.80 (s, 1H), 7.40 (d, $J=3.0$ $\mathrm{Hz}, 1 \mathrm{H}), 7.01$ (d, $J=3.0 \mathrm{~Hz}, 1 \mathrm{H}), 3.80$ (s, 3H); ${ }^{13} \mathrm{C}$ NMR (75 MHz, $\mathrm{CDCl}_{3}$ ): $\delta$ 196.0, 153.1, 152.7, 127.6, 120.6, 116.0, 111.7, 56.3; IR (neat) $v_{\max }$ 3072, 1654, 1611, 1456, 1321, $1133 \mathrm{~cm}^{-1}$; CIMS ( $\mathrm{NH}_{3}$ gas): 229.9, 152.0, 53.0; HRMS (CI, $\mathrm{NH}_{3}$ ) m/z calcd for $\mathrm{C}_{8} \mathrm{H}_{8} \mathrm{BrO}_{3}[\mathrm{M}]^{+}$229.958, found 229.995 .

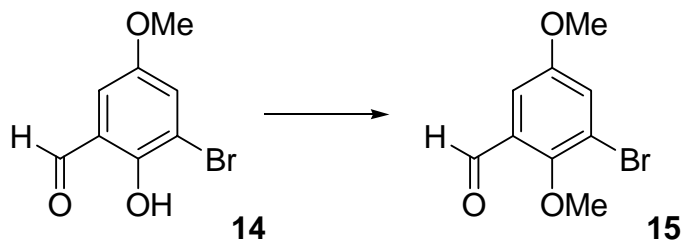

3-Bromo-2,5-dimethoxy-benzaldehyde 15. To a solution of phenol 14 (12.0 g, $51.9 \mathrm{mmol})$ in DMF (210 mL) was added potassium carbonate $(10.0 \mathrm{~g}, 72.3 \mathrm{mmol})$ and dimethylsulfate (6.9 $\mathrm{mL}, 72.9 \mathrm{mmol})$. The resulting purple mixture was allowed to stir at rt for 24 hours at which time water was added. The organic layer was separated and the aqueous layer was extracted with AcOEt. The combined organic extracts were washed with brine, dried over $\mathrm{MgSO}_{4}$, filtered and concentrated. The crude residue was purified by flash chromatography over silica gel (hexanes/AcOEt: 8/2) to yield a white powder (12.6 g, $51.4 \mathrm{mmol}, 99 \%$ ). Mp : $68{ }^{\circ} \mathrm{C} ;{ }^{1} \mathrm{H}$ NMR 
(400 MHz, $\mathrm{CDCl}_{3}$ ): $\delta 10.29$ (s, 1H), 7.36 (d, $\left.J=3.0 \mathrm{~Hz}, 1 \mathrm{H}\right), 7.25$ (d, $\left.J=3.0 \mathrm{~Hz}, 1 \mathrm{H}\right), 3.91$ (s, 3H), 3.79 (s, 3H); ${ }^{13} \mathrm{C}$ NMR (75 MHz, $\left.\mathrm{CDCl}_{3}\right): \delta 188.8,156.3,154.2,130.4,126.2,118.4,110.0$, 63.5, 55.7; IR (neat) $v_{\max }$ 2960, 2865, 1689, $1474 \mathrm{~cm}^{-1}$; CIMS ( $\mathrm{NH}_{3}$ gas): 245.9, 228.9, 199.9, 172.9, 79.0; HRMS (CI, $\mathrm{NH}_{3}$ ) m/z calcd for $\mathrm{C}_{9} \mathrm{H}_{10} \mathrm{BrO}_{3}[\mathrm{M}+\mathrm{H}]^{+}$244.981, found 244.984 .

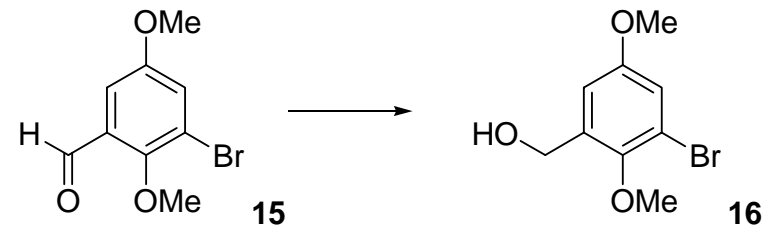

3-Bromo-2,5-dimethoxy-4-hydroxymethylbenzene 16. To a solution of aldehyde 15 (9.3 g, $37.9 \mathrm{mmol})$ in THF $(130 \mathrm{~mL})$ was slowly added $\mathrm{BH}_{3}$ THF (1M solution in THF, $42 \mathrm{~mL}, 42$ $\mathrm{mmol})$ at $0^{\circ} \mathrm{C}$. The resulting mixture was then stirred at $0^{\circ} \mathrm{C}$ for 1 hour at which time $25 \mathrm{~mL}$ of $\mathrm{MeOH}$ were added dropwise. After evaporation of the solvents, the residue was dissolved in ether and water was added. The organic layer was separated and the aqueous layer was extracted with ether. The combined organic extracts were washed with brine, dried over $\mathrm{MgSO}_{4}$, filtered and concentrated to give a white powder (9.3 g, $37.9 \mathrm{mmol}$, quant.) used without further purification for the next step. Mp : $69{ }^{\circ} \mathrm{C} ;{ }^{1} \mathrm{H}$ NMR $\left(400 \mathrm{MHz}, \mathrm{CDCl}_{3}\right): \delta 6.96(\mathrm{~d}, J=2.8 \mathrm{~Hz}$, 1H), 6.85 (d, $J=2.8 \mathrm{~Hz}, 1 \mathrm{H}), 4.64$ (d, $J=6.0 \mathrm{~Hz}, 2 \mathrm{H}), 3.77$ (s, 3H), 3.73 (s, 3H), 2.56 (t, $J=6.0$ $\mathrm{Hz}, 1 \mathrm{H}) ;{ }^{13} \mathrm{C} \mathrm{NMR}\left(75 \mathrm{MHz}, \mathrm{CDCl}_{3}\right): \delta$ 156.6, 148.6, 136.5, 117.6, 117.3, 113.7, 61.4, 61.1, 55.8; IR (neat) $v_{\max }$ 3397, 2938, 1477, 1226, $1050 \mathrm{~cm}^{-1}$; CIMS ( $\mathrm{NH}_{3}$ gas): 246.1, 233.1, 231.1, 229.1, 124.1, 109.1; HRMS (CI, NH${ }_{3}$ ) m/z calcd for $\mathrm{C}_{9} \mathrm{H}_{11} \mathrm{BrO}_{3}[\mathrm{M}]^{+}$245.989, found 245.991.

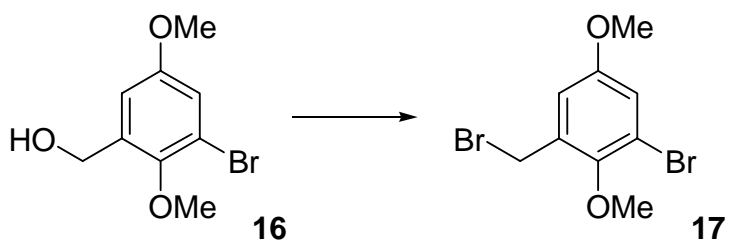

1-Bromo-3-bromomethyl-2,5-dimethoxybenzene 17. To a solution of alcohol 16 (5.6 g, 23.9 $\mathrm{mmol})$ in THF $(140 \mathrm{~mL})$ were added at $0^{\circ} \mathrm{C}$ triphenylphosphine $(9.4 \mathrm{~g}, 35.8 \mathrm{mmol})$ and carbon 
tetrabromide (11.9 g, $35.9 \mathrm{mmol})$. The resulting mixture was then stirred at $0^{\circ} \mathrm{C}$ until complete dissolution of the reagents (typically 10 minutes) and for 1 hour at rt. The white precipitate was removed by filtration over a plug of Celite ${ }^{\circledR}$ and the filtrate was diluted with ether $(400 \mathrm{~mL})$, washed with water $(200 \mathrm{~mL})$ and brine $(200 \mathrm{~mL})$, dried over $\mathrm{MgSO}_{4}$, filtered and concentrated. After removing of the excess of carbon tetrabromide under high vacuum, the residue was purified by chromatography over silica gel (hexanes/AcOEt: 8/2) to yield a pale yellow oil (7.1 g, 22.9 mmol, 96 \%). ${ }^{1} \mathrm{H}$ NMR (400 MHz, $\mathrm{CDCl}_{3}$ ): $\delta 7.03$ (br. s, $1 \mathrm{H}$ ), 6.85 (br. s, 3H), 4.50 (s, 2H), 3.90 (s, 3H), 3.75 (s, 3H); ${ }^{13} \mathrm{C}$ NMR (75 MHz, $\left.\mathrm{CDCl}_{3}\right): \delta 156.3,149.3,133.5,119.5,117.8$, 115.8, 61.6, 55.9, 27.8; IR (neat) $v_{\max }$ 2957, 2833, 1479, 1229, $1047 \mathrm{~cm}^{-1}$; CIMS (NH $\mathrm{NH}_{3}$ gas: 310.1, 231.1, 229.1, 150.1, 91.1; HRMS (CI, $\mathrm{NH}_{3}$ ) m/z calcd for $\mathrm{C}_{9} \mathrm{H}_{11} \mathrm{Br}_{2} \mathrm{O}_{2}[\mathrm{M}+\mathrm{H}]^{+} 308.913$, found 308.912 .

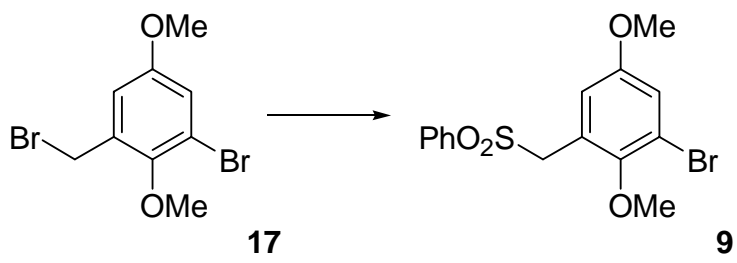

1-Bromo-3-benzenesulfonylmethyl-2,5-dimethoxybenzene 9. To a solution of bromide 17 (4.0 g, $12.9 \mathrm{mmol}$ ) in DMF (60 mL) was added sodium benzenesulfinate (2.35 g, $14.3 \mathrm{mmol}$ ). The resulting mixture was then stirred at rt for 24 hours and subsequently diluted with water. The organic layer was separated and the aqueous layer was extracted with AcOEt. The combined organic extracts were washed with brine, dried over $\mathrm{MgSO}_{4}$, filtered and concentrated. The crude residue was purified by flash chromatography over silica gel (hexanes/AcOEt: 7/3) to yield a white solid (4.1 g, $11.4 \mathrm{mmol}, 86$ \%). Mp : $104{ }^{\circ} \mathrm{C} ;{ }^{1} \mathrm{H}$ NMR (400 MHz, $\mathrm{CDCl}_{3}$ ): $\delta 7.75$ (d, $J=$ $7.8 \mathrm{~Hz}, 2 \mathrm{H}), 7.62$ (t, $J=7.8 \mathrm{~Hz}, 1 \mathrm{H}), 7.50$ (t, $J=7.8 \mathrm{~Hz}, 2 \mathrm{H}), 7.05$ (d, $J=3.2 \mathrm{~Hz}, 1 \mathrm{H}), 6.74$ (dt, $J=3.2 \mathrm{~Hz}, 1 \mathrm{H}), 4.33$ (s, 2H), 3.59 (s, 3H), 3.58 (s, 3H); ${ }^{13} \mathrm{C}$ NMR (75 MHz, $\mathrm{CDCl}_{3}$ ): $\delta 155.6$, 150.1, 138.5, 134.0, 129.1, 128.5, 123.5, 120.0, 117.3, 115.9, 61.6, 56.5, 55.6; IR (neat) $v_{\max }$ 3004, 2941, 2836, 1480, 1308, 1228, 1156, 1085, $997 \mathrm{~cm}^{-1}$; CIMS ( $\mathrm{NH}_{3}$ gas): 372.2, 248.1, 246.1, 231.1, 229.1, 150.1, 125.0, 109.9; HRMS (CI, $\left.\mathrm{NH}_{3}\right) \mathrm{m} / \mathrm{z}$ calcd for $\mathrm{C}_{15} \mathrm{H}_{15} \mathrm{BrO}_{4} \mathrm{~S}[\mathrm{M}]^{+}$ 369.987, found 369.990 . 


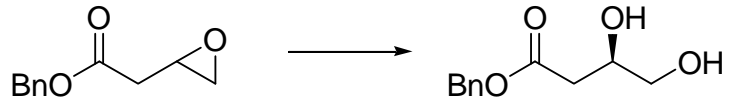

$( \pm)-18$

19

(R)-3,4-Dihydroxy-butyric acid benzyl ester 19. A $25 \mathrm{~mL}$ flask was charged with ( \pm )-benzyl3,4-epoxybutyrate ${ }^{\mathrm{S} 1}$ (12.0 g, $\left.62.4 \mathrm{mmol}\right)$ and $(S, S)$-Co(salen) ${ }^{\mathrm{S} 2}$ (377 mg, $\left.0.62 \mathrm{mmol}\right)$. Acetic acid $(144 \mu \mathrm{L}, 2.49 \mathrm{mmol})$ and THF $(620 \mu \mathrm{L})$ were added. The reaction flask was cooled to $0^{\circ} \mathrm{C}$ and water ( $620 \mu \mathrm{L}, 34.4 \mathrm{mmol}$ ) was added in one portion. The reaction mixture was allowed to warm to rt and monitored by ${ }^{1} \mathrm{H}$ NMR: when the reaction reached $49 \%$ conversion (typically 4-6 hours), the crude mixture was loaded on silica gel (hexanes/AcOEt: 7/3 to recover the unreacted enantiomer of the epoxyde then AcOEt) to yield the desired diol as a brown oil (6.2 g, 29.5 mmol, $47 \%$ ). The ee of this product was determined to be $99 \%$ by chiral HPLC analysis (Chiracel OD, hexanes/iPrOH: 95/5, 1 mL.min ${ }^{-1}, t_{R}$ (minor) 38.3 min, $t_{R}$ (major) $41.7 \mathrm{~min}$ ). $[\alpha]_{\mathrm{D}}^{20}+15.1$ (c 1.29, MeOH); lit. ${ }^{\mathrm{S}}[\alpha]_{\mathrm{D}}^{20}+14\left(c\right.$ 2, MeOH); ${ }^{1} \mathrm{H}$ NMR $\left(400 \mathrm{MHz}, \mathrm{CDCl}_{3}\right): \delta 7.24-$ 7.27 (m, 5H), 5.04 (s, 2H), 3.98-4.06 (m, 1H), 3.55 (ddd, $J=3.6,6.2,10.8 \mathrm{~Hz}, 1 \mathrm{H}$ ), 3.41 (ddd, $J$ = 6.2, 6.4, $10.8 \mathrm{~Hz}, 1 \mathrm{H}$ ), 3.15 (d, $J=3.6 \mathrm{~Hz}, 1 \mathrm{H}), 2.49$ (dd, $J=8.8,16.8 \mathrm{~Hz}, 1 \mathrm{H}$ ), 2.42 (dd, $J=$ 4.0, $16.8 \mathrm{~Hz}, 1 \mathrm{H}), 2.12(\mathrm{t}, J=6.0 \mathrm{~Hz}, 1 \mathrm{H}) ;{ }^{13} \mathrm{C} \mathrm{NMR}\left(75 \mathrm{MHz}, \mathrm{CDCl}_{3}\right): \delta 172.4,135.7,128.7$, 128.4, 128.3, 68.7, 66.6, 65.7, 37.8; IR (neat) $v_{\max }$ 3395, 2940, 1732, 1167, $1043 \mathrm{~cm}^{-1}$; CIMS ( $\mathrm{NH}_{3}$ gas): 228.1, 211.0, 181.1, 108.0, $91.0 \mathrm{~cm}^{-1}$; HRMS (CI, $\mathrm{NH}_{3}$ ) $\mathrm{m} / \mathrm{z}$ calcd for $\mathrm{C}_{11} \mathrm{H}_{15} \mathrm{O}_{4}$ $[\mathrm{M}+\mathrm{H}]^{+}$211.097, found 211.095.

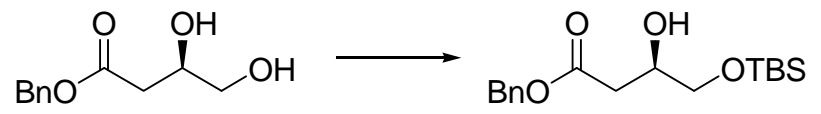

19

(R)-4-(tert-Butyl-dimethylsilyloxy)-3-hydroxy-butyric acid benzyl ester. To a solution of diol 19 (9.6 g, $45.7 \mathrm{mmol})$ in THF (50 mL) were added imidazole (4.1 g, $60 \mathrm{mmol})$ and TBSCl (7.4

\footnotetext{
${ }^{\mathrm{S} 1}$ Hoff, B. G.; Anthonsen, T. Tetrahedron: Asymmetry 1999, 10, 1401-1412

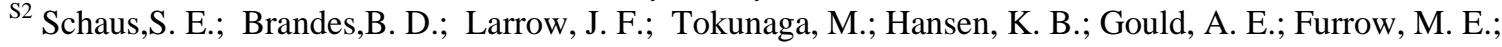
Jacobsen, E. N. J. Am. Chem. Soc. 2002, 124, 1307-1315

${ }^{\text {S3 }}$ Bellamy, F. D.; Bondoux, M.; Dodey, P. Tetrahedron Lett. 1990, 31, 7323-7326
} 
g, $49.1 \mathrm{mmol})$. The resulting slurry was stirred at $0^{\circ} \mathrm{C}$ for 90 minutes and water was added. The organic layer was separated and the aqueous layer was extracted with AcOEt. The combined organic extracts were washed with brine, dried over $\mathrm{MgSO}_{4}$, filtered and concentrated. The crude residue was purified by flash chromatography over silica gel (hexanes/AcOEt: 8/2) to yield a colorless oil (14.3 g, $44.1 \mathrm{mmol}, 96 \%)$. $[\alpha]_{\mathrm{D}}^{20}+10.3$ (c 0.68, $\left.\mathrm{CHCl}_{3}\right) ;{ }^{1} \mathrm{H}$ NMR (400 MHz, $\left.\mathrm{CDCl}_{3}\right): \delta 7.31-7.40(\mathrm{~m}, 5 \mathrm{H}), 5.17(\mathrm{~s}, 2 \mathrm{H}), 4.05-4.13(\mathrm{~m}, 1 \mathrm{H}), 3.61$ (A of ABX, $J=6.2,10.8 \mathrm{~Hz}$, 1H), 3.56 (B of ABX, $J=5.3,10.8 \mathrm{~Hz}, 1 \mathrm{H}$ ), 2.80 (d, $J=5.5 \mathrm{~Hz}, 1 \mathrm{H}), 2.57$ (d, $J=7.2 \mathrm{~Hz}, 2 \mathrm{H}$ ), 0.88 (s, 9H), 0.02 (s, 6H); ${ }^{13} \mathrm{C}$ NMR (75 MHz, $\left.\mathrm{CDCl}_{3}\right): \delta 172.2,135.9,128.8,128.6,128.5,68.7$, 66.6, 66.2, 38.1, 25.9, 18.3, -5.4; IR (neat) $v_{\max }$ 3469, 2929, 2857, 1737, 1121, $838 \mathrm{~cm}^{-1}$; CIMS ( $\mathrm{NH}_{3}$ gas): 325.1, 307.1, 217.1, 117.1, 91.0; HRMS (CI, $\mathrm{NH}_{3}$ ) m/z calcd for $\mathrm{C}_{17} \mathrm{H}_{28} \mathrm{SiO}_{4}[\mathrm{M}]^{+}$ 324.176, found 324.175.

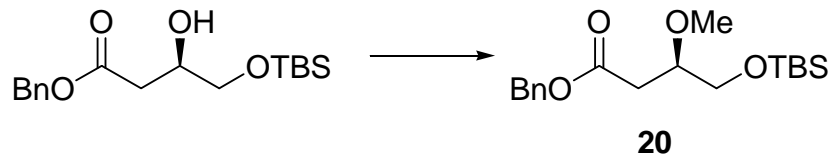

(R)-4-(tert-Butyl-dimethylsilyloxy)-3-methoxy-butyric acid benzyl ester 20. To a solution of (R)-4-(tert-Butyl-dimethylsilyloxy)-3-hydroxy-butyric acid benzyl ester (12.8 g, $39.4 \mathrm{mmol})$ in

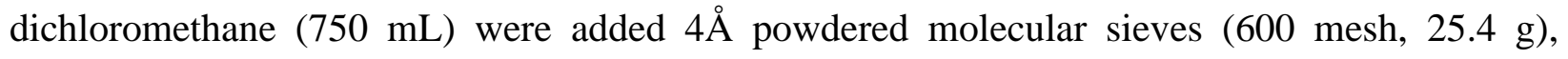
Proton-Sponge $^{\circledR}(25.4 \mathrm{~g}, 118.5 \mathrm{mmol})$ and trimethyloxonium tetrafluoroborate (14.6 g, 98.7 mmol). The resulting mixture was stirred at rt for 2 hours, filtrated over a plug of Celite ${ }^{\circledR}$ (rinsed with dichloromethane), washed successively with 1M CuSO4 and brine, dried over $\mathrm{MgSO}_{4}$, filtered and concentrated. The crude residue was purified by flash chromatography over silica gel (hexanes/AcOEt: 95/5) to yield a colorless oil (12.8 g, $37.8 \mathrm{mmol}, 96 \%)$. $[\alpha]_{\mathrm{D}}^{20}+12.9(c 0.51$, $\left.\mathrm{CHCl}_{3}\right) ;{ }^{1} \mathrm{H} \mathrm{NMR}\left(400 \mathrm{MHz}, \mathrm{CDCl}_{3}\right): \delta 7.30-7.35$ (m, 5H), $5.13(\mathrm{ABq}, J=12.4 \mathrm{~Hz}, 2 \mathrm{H}), 3.66-$ 3.74 (m, 2H), 3.56 (dd, $J=5.4,10.2 \mathrm{~Hz}, 1 \mathrm{H}$ ), 3.37 (s, 3H), 2.62 (dd, $J=4.6,15.8 \mathrm{~Hz}, 1 \mathrm{H}), 2.50$ (dd, $J=7.8,15.8 \mathrm{~Hz}, 1 \mathrm{H}), 0.87$ (s, 9H), 0.03 (s, 6H); ${ }^{13} \mathrm{C}$ NMR (75 $\left.\mathrm{MHz}, \mathrm{CDCl}_{3}\right): \delta 171.9$, 136.2, 128.8, 128.5, 128.4, 78.5, 66.4, 64.2, 58.2, 37.2, 25.9, 18.3, -5.4; IR (neat) $v_{\max } 2929$, 2857, 1738, 1462, 1161, $837 \mathrm{~cm}^{-1}$; CIMS ( $\mathrm{NH}_{3}$ gas): 339.4, 281.3, 231.3, 181.2, 131.1, 91.1; HRMS (CI, $\mathrm{NH}_{3}$ ) m/z calcd for $\mathrm{C}_{18} \mathrm{H}_{31} \mathrm{SiO}_{4}[\mathrm{M}+\mathrm{H}]^{+}$339.199, found 339.194. 


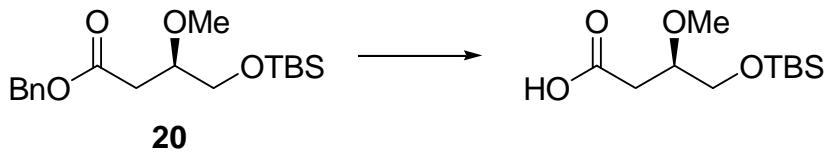

(R)-4-(tert-Butyl-dimethylsilyloxy)-3-methoxybutyric acid. A solution of benzyl ester 20 (12.6 g, $37.2 \mathrm{mmol})$ in methanol $(700 \mathrm{~mL})$ was treated with Palladium on carbon (10 \% wt. on activated carbon, $1.8 \mathrm{~g}$ ) and stirred under an atmosphere of hydrogen for 90 minutes. The mixture was filtrated over a plug of Celite ${ }^{\circledR}$ and concentrated to yield a colorless oil used in the next step without further purification (9.2 g, $37.1 \mathrm{mmol}$, quant.). $[\alpha]_{\mathrm{D}}^{20}+16.6$ (c 0.71, $\mathrm{CHCl}_{3}$ ); ${ }^{1} \mathrm{H}$ NMR (400 MHz, $\mathrm{CDCl}_{3}$ ): $\delta$ 3.66-3.72 (m, 2H), 3.59 (dd, $\left.J=4.4,9.2 \mathrm{~Hz}, 1 \mathrm{H}\right), 3.43$ (s, 3H), 2.65 (dd, $J=3.8,15.8 \mathrm{~Hz}, 1 \mathrm{H}$ ), 2.52 (dd, $J=6.8,15.8 \mathrm{~Hz}, 1 \mathrm{H}), 0.85$ (s, 9H), 0.04 (s, 6H); ${ }^{13} \mathrm{C}$ NMR (75 MHz, $\mathrm{CDCl}_{3}$ ): $\delta 177.4,78.3,64.0,58.1,37.0,25.9,25.7,18.3$, -5.5; IR (neat) $v_{\max }$ 3100, 2930, 2858, 1713, 1119, $838 \mathrm{~cm}^{-1}$; CIMS ( $\mathrm{NH}_{3}$ gas): 249.2, 231.2, 215.2, 191.2, 159.1, 131.1, 115.1, 89.0, 75.1; HRMS (CI, $\left.\mathrm{NH}_{3}\right) \mathrm{m} / \mathrm{z}$ calcd for $\mathrm{C}_{11} \mathrm{H}_{25} \mathrm{SiO}_{4}[\mathrm{M}+\mathrm{H}]^{+}$249.152, found 249.151.

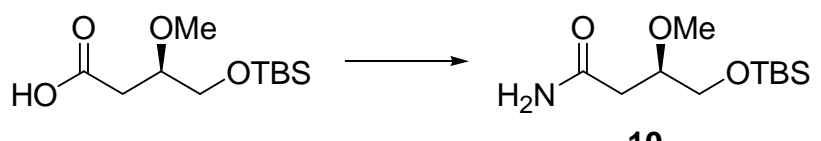

10

(R)-4-(tert-Butyl-dimethylsilyloxy)-3-methoxybutyramide 10. To a stirred solution of acid (R)-4-(tert-Butyl-dimethylsilyloxy)-3-methoxybutyric $\quad$ acid $\quad(8.8 \quad \mathrm{~g}, \quad 35.4 \mathrm{mmol})$ in dichloromethane $(525 \mathrm{~mL})$ were added triethylamine $(10.5 \mathrm{~mL}, 74.7 \mathrm{mmol})$ and ethyl chloroformate $(3.9 \mathrm{~mL}, 40.8 \mathrm{mmol})$ at $-20^{\circ} \mathrm{C}$. The resulting mixture was kept under vigorous stirring and after $40 \mathrm{~min}$, a slow stream of ammonia was passed through the mixture for $10 \mathrm{~min}$ (until TLC showed complete disappearance of the mixed anhydride) whereby the temperature was carefully kept bellow $-10^{\circ} \mathrm{C}$. Reaction was quenched with water, the organic layer was separated and the aqueous layer was extracted with dichloromethane. The combined organic extracts were washed with $1 \mathrm{M} \mathrm{HCl}$ and brine, dried over $\mathrm{MgSO}_{4}$, filtered and concentrated. The crude residue was purified by flash chromatography over a short plug of silica gel (eluting with 
AcOEt) to yield a white oily solid (8.0 g, $32.3 \mathrm{mmol}, 91 \%) .[\alpha]_{\mathrm{D}}^{20}+31.7$ (c 0.46, $\left.\mathrm{CHCl}_{3}\right) ;{ }^{1} \mathrm{H}$ NMR (400 MHz, $\mathrm{CDCl}_{3}$ ): $\delta 6.28$ (br. s, 1H), 6.21 (br. s, 1H), 3.41-3.62 (m, 3H), 3.36 (s, 3H), 2.37 (dd, $J=3.2,15.2 \mathrm{~Hz}, 1 \mathrm{H}), 2.31$ (dd, $J=6.8,15.2 \mathrm{~Hz}, 1 \mathrm{H}), 0.81$ (s, 9H), -0.02 (s, 6H); ${ }^{13} \mathrm{C}$ NMR (75 MHz, $\mathrm{CDCl}_{3}$ ): $\delta 174.3,78.8,64.1,57.8,38.3,25.8,18.2,-5.6$; IR (neat) $v_{\max } 3344$, 3200, 2930, 2858, 1670, 1118, $838 \mathrm{~cm}^{-1}$; CIMS ( $\mathrm{NH}_{3}$ gas): 248.1, 231.1, 229.1, 190.2, 150.1, 124.1, 91.1; HRMS (CI, $\mathrm{NH}_{3}$ ) m/z calcd for $\mathrm{C}_{11} \mathrm{H}_{25} \mathrm{NSiO}_{3}[\mathrm{M}+\mathrm{H}]^{+}$248.168, found 248.171.

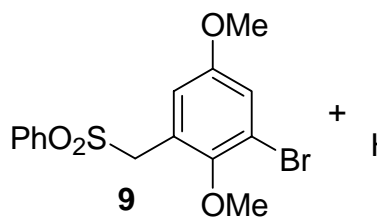

OMe
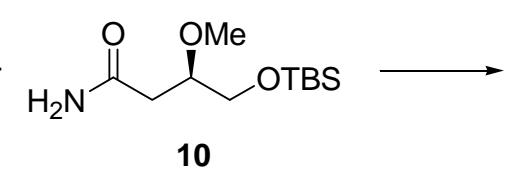

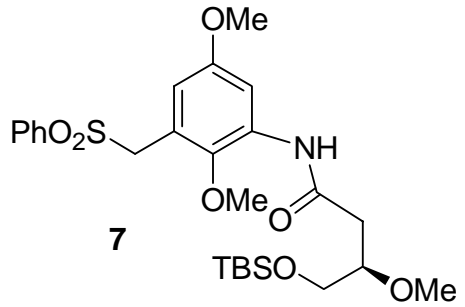

(R)-N-(3-Benzenesulfonylmethyl-2,5-dimethoxy-phenyl)-4-(tert-butyl-dimethylsilyloxy)-3-

methoxy-butyramide 7. In a $350 \mathrm{~mL}$ pressure tube were dissolved aryl bromide 9 (12.0 g, 32.3 mmol), amide 10 (8.0 g, $32.3 \mathrm{mmol}$ ) and potassium carbonate (powder, 325 mesh, 9.0 g, 65.1 mmol) in toluene $(60 \mathrm{~mL})$. Copper(I) iodide $(1.22 \mathrm{~g}, 6.4 \mathrm{mmol})$ and $N, N^{\prime}-$ dimethylethylenediamine $(1.35 \mathrm{~mL}, 12.7 \mathrm{mmol})$ were then added, the pressure tube was closed and the blue suspension was heated to $110^{\circ} \mathrm{C}$ for 36 hours. Crude reaction mixture was then filtrated over a plug of silica gel (washed with AcOEt) and concentrated. The crude residue was purified by flash chromatography over silica gel (hexanes/AcOEt: 7/3) to yield a white solid (14.3 g, $26.6 \mathrm{mmol}, 82 \%) . \mathrm{Mp}: 84{ }^{\circ} \mathrm{C}$; $[\alpha]_{\mathrm{D}}^{20}-9.4$ (c 1.04, $\left.\mathrm{CHCl}_{3}\right) ;{ }^{1} \mathrm{H} \mathrm{NMR}\left(400 \mathrm{MHz}, \mathrm{CDCl}_{3}\right)$ : $\delta 8.56$ (s, 1H), 7.97 (d, $J=2.8 \mathrm{~Hz}, 1 \mathrm{H}), 7.71$ (d, $J=7.2 \mathrm{~Hz}, 2 \mathrm{H}), 7.58$ (t, $J=7.8 \mathrm{~Hz}, 1 \mathrm{H}), 7.45$ (t, $J=7.8 \mathrm{~Hz}, 2 \mathrm{H}), 6.32$ (d, $J=3.2 \mathrm{~Hz}, 1 \mathrm{H}), 4.34$ (s, 2H), 3.62-3.70 (m, 6H), 3.57 (s, 3H), 3.43 (s, 3H), 2.60 (dd, $J=2.6,15.4 \mathrm{~Hz}, 1 \mathrm{H}), 2.51$ (dd, $J=7.2,15.4 \mathrm{~Hz}, 1 \mathrm{H}), 0.85$ (s, 9H), 0.02 (s, $6 \mathrm{H}$ ); ${ }^{13} \mathrm{C}$ NMR (75 MHz, $\left.\mathrm{CDCl}_{3}\right): \delta 169.8,156.0,142.2$, 138.6, 133.9, 132.8, 129.1, 128.9, 121.5, 111.1, 107.6, 78.8, 63.8, 61.8, 57.9, 56.5, 55.6, 40.2, 25.8, 18.3, -5.5; IR (neat) $v_{\max } 3321$, 2930, 2857, 1687, 1533, 1157, $838 \mathrm{~cm}^{-1}$; CIMS ( $\mathrm{NH}_{3}$ gas): 538.4, 480.2, 398.3, 340.2, 308.1, 231.1, 166.1, 151.1; HRMS (CI, $\mathrm{NH}_{3}$ ) m/z calcd for $\mathrm{C}_{26} \mathrm{H}_{40} \mathrm{NSiSO}_{7}[\mathrm{M}+\mathrm{H}]^{+}$538.229, found 538.228. 


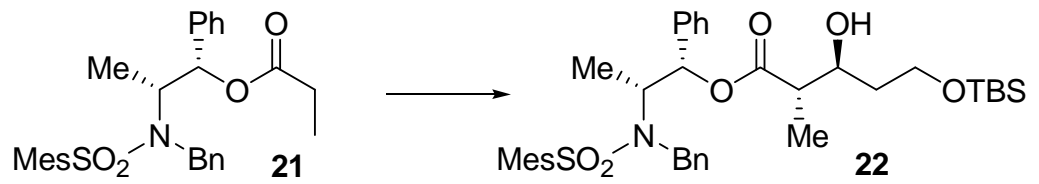

[1(1S,2R),2S,3S]-5-(tert-butyl-dimethylsilyloxy)-3-hydroxy-2-methyl-pentanoic acid 2-(Nbenzyl- $N$-mesitylenesulfonyl)amino-1-phenyl-propyl ester 22. An oven-dried $250 \mathrm{~mL}$ round bottom flask equipped with a stir bar is charged with (1R,2S)-2-( $N$-benzyl- $N$ mesitylenesulfonyl)amino-1-phenyl-propyl propionate $\mathbf{2 1}^{\mathrm{S} 4}$ (14.7 g, $\left.30.7 \mathrm{mmol}\right)$ and flushed with $\mathrm{N}_{2}$. The material was dissolved in $155 \mathrm{~mL}$ dichloromethane, and $\mathrm{Et}_{3} \mathrm{~N}(9.8 \mathrm{~mL}, 67.5 \mathrm{mmol}$ ) was added to the reaction flask. The solution was cooled to $-78{ }^{\circ} \mathrm{C}$ and a solution of dicyclohexylborontriflate $^{\mathrm{S} 5}(1.0 \mathrm{M}$ solution in hexanes, $77.0 \mathrm{~mL}, 77.0 \mathrm{mmol})$ was added dropwise. The resulting solution was allowed to stir at $-78{ }^{\circ} \mathrm{C}$ for 2 hours, and tertbutyldimethylsilyloxypropionaldehyde ${ }^{\mathrm{S} 6}(7.0 \mathrm{~g}, 36.8 \mathrm{mmol})$ was added dropwise. The reaction mixture was stirred for 1 hour at $-78{ }^{\circ} \mathrm{C}$ then 1 hour at room temperature, at which time is was quenched by addition of pH7 buffer $(130 \mathrm{~mL})$. The mixture was diluted with $\mathrm{MeOH}(130 \mathrm{~mL})$ and hydrogen peroxide (30 wt. \% solution, $65 \mathrm{~mL}$ ) was carefully added. The resulting mixture was vigorously stirred overnight and was then concentrated. The residue was partitioned between $\mathrm{H}_{2} \mathrm{O}(400 \mathrm{~mL})$, and $\mathrm{CH}_{2} \mathrm{Cl}_{2}(200 \mathrm{~mL})$. The aqueous layer was extracted with $\mathrm{CH}_{2} \mathrm{Cl}_{2}(3 \times 150$ $\mathrm{mL})$. The combined organic extracts were washed brine $(250 \mathrm{~mL})$, dried over $\mathrm{MgSO}_{4}$, filtered and concentrated. The crude residue was purified by flash chromatography over silica gel (hexanes/AcOEt: 8/2) to yield a white foam (19.3 g, $28.9 \mathrm{mmol}, 94 \%$ ). The de of this reaction was determined to be higher to $30: 1$ by ${ }^{1} \mathrm{H}$ NMR analysis of the crude reaction mixture. $[\alpha]_{D}^{20}$ 13.4 (c 1.03, $\mathrm{CHCl}_{3}$ ); ${ }^{1} \mathrm{H} \mathrm{NMR}\left(400 \mathrm{MHz}, \mathrm{CDCl}_{3}\right): \delta 7.31$ (d, $\left.J=7.2 \mathrm{~Hz}, 2 \mathrm{H}\right), 7.12-7.21$ (m, 7H), 6.86 (s, 2H), 6.81 (dd, $J=1.2,7.2 \mathrm{~Hz}, 2 \mathrm{H}), 5.77$ (d, $J=3.6 \mathrm{~Hz}, 1 \mathrm{H}), 4.80$ (A of ABq, $J=$ $16.8 \mathrm{~Hz}, 1 \mathrm{H}$ ), 4.58 (B of ABq, $J=16.8 \mathrm{~Hz}, 1 \mathrm{H}), 4.03-4.06$ (m, 1H); 3.90-3.97 (m, 1H); 3.803.86 (m, 1H); 3.72-3.78 (m, 1H), 3.56 (br. s, 1H), 2.48-2.54 (m, 1H), 2.48 (s, 6H), 2.26 (s, 3H), 1.60-1.64 (m, 2H), 1.13 (d, $J=6.8 \mathrm{~Hz}, 3 \mathrm{H}), 1.08$ (d, $J=7.6 \mathrm{~Hz}, 3 \mathrm{H}), 0.86$ (s, 9H), 0.04-0.06 (m, $6 \mathrm{H}) ;{ }^{13} \mathrm{C}$ NMR (75 MHz, $\mathrm{CDCl}_{3}$ ): $\delta$ 173.9; 142.2; 140.2; 138.7; 138.4; 132.0; 128.2; 128.1;

\footnotetext{
${ }^{\mathrm{S} 4}$ Abiko, A. Organic Syntheses 2002, 79, 109-115

${ }^{5} 5$ Abiko, A. Organic Syntheses 2002, 79, 103-108

${ }^{\text {s6 }}$ Groth, T., Meldal, M. J. Comb. Chem. 2001, 3, 34-44
} 
127.7; 127.5; 127.2; 126.9; 125.8; 77.9; 72.9; 61.9; 56.6; 48.0; 45.6; 35.0; 25.5; 22.6; 20.6; 13.0; 12.9; -5.9; IR (neat) $v_{\max } 3484 ; 3034 ; 2931 ; 2857 ; 1740 ; 1604 \mathrm{~cm}^{-1}$; CIMS (NH3 gas): 496.4; 406.4; 316.2; 22.2; 119.1; HRMS (CI, $\left.\mathrm{NH}_{3}\right) \mathrm{m} / \mathrm{z}$ calcd for $\mathrm{C}_{37} \mathrm{H}_{54} \mathrm{NO}_{6} \mathrm{SSi}[\mathrm{M}+\mathrm{H}]^{+}$668.344, found: 668.341.

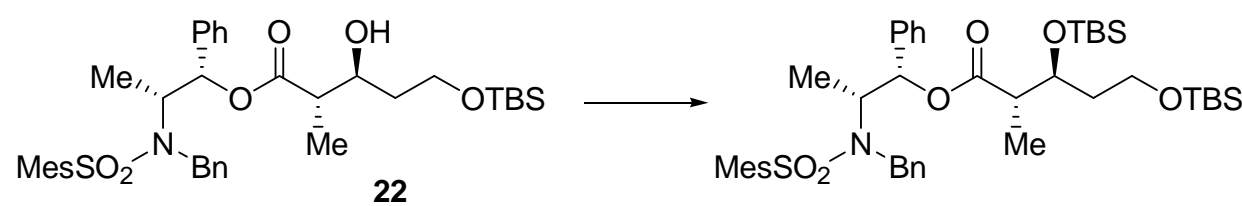

[1(1S,2R),2S,3S]-3,5-Bis-(tert-butyl-dimethylsilyloxy)-2-methyl-pentanoic acid 2-( $N$-benzyl$N$-mesitylenesulfonyl)amino]-1-phenyl-propyl ester. To a solution of alcohol 22 (11.5 g, 17.2 mmol) and 2,6-lutidine $(6.0 \mathrm{~mL}, 51.5 \mathrm{mmol})$ in dichloromethane $(130 \mathrm{~mL})$ was added TBSOTf $(8.0 \mathrm{~mL}, 34.8 \mathrm{mmol})$ at $0{ }^{\circ} \mathrm{C}$. The resulting solution was warmed to rt over 1 hour and quenched with a saturated solution of $\mathrm{NaHCO}_{3}$. The organic layer was separated and the aqueous layer was extracted with dichloromethane. The combined organic extracts were washed with $1 \mathrm{M} \mathrm{HCl}$, saturated solution of $\mathrm{NaHCO}_{3}$ and brine, dried over $\mathrm{MgSO}_{4}$, filtered and concentrated. The crude residue was purified by flash chromatography over silica gel (hexanes/AcOEt: 95/5) to yield a colorless oil (12.9 g, $16.5 \mathrm{mmol}, 96 \%$ \% $[\alpha]_{\mathrm{D}}^{20}-16.8$ (c 1.13, $\mathrm{CHCl}_{3}$ ); ${ }^{1} \mathrm{H} \mathrm{NMR}(400 \mathrm{MHz}$, $\left.\mathrm{CDCl}_{3}\right): \delta 7.21$ (d, $\left.\left.J=6.4 \mathrm{~Hz}, 2 \mathrm{H}\right), 6.98-7.15(\mathrm{~m}, 7 \mathrm{H}), 6.72(\mathrm{~s}, 2 \mathrm{H}), 6.71 \mathrm{~d}, J=7.6 \mathrm{~Hz}, 2 \mathrm{H}\right)$, 5.61 (d, $J=5.2 \mathrm{~Hz}, 1 \mathrm{H}), 4.65$ (A of ABq, $J=16.4 \mathrm{~Hz}, 1 \mathrm{H}), 4.38$ (B of ABq, $J=16.4 \mathrm{~Hz}, 1 \mathrm{H}$ ), 3.91-3.98 (m, 2H); 3.41-3.52 (m, 2H), 2.43 (qd, $J=4.8,6.8$ Hz, 1H), 2.31 (s, 6H), 2.16 (s, 3H), 1.58-1.63 (m, 1H), 1.47 (quint., $J=6.8 \mathrm{~Hz}, 1 \mathrm{H}), 1.30-1.36$ (m, 1H), 1.13-1.19 (m, 1H), 1.6 (d, $J$ = 7.2 Hz, 3H), 0.94 (d, J=7.2 Hz, 3H), 0.74 (s, 9H), 0.72 (s, 9H), -0.08 (s, 6H), -0.15 (s, 3H), 0.16 (s, 3H); ${ }^{13} \mathrm{C}$ NMR (75 MHz, $\left.\mathrm{CDCl}_{3}\right): \delta 172.6,142.7,140.6,138.7,138.5,133.4,132.3$, 128.6, 128.5, 128.4, 128.1, 127.4, 126.6, 78.0, 70.2, 59.6, 59.8, 48.2, 45.5, 36.1, 36.0, 25.9, 24.1, 22.9, 20.9, 18.2, 18.1, 14.2, 11.4, -4.6, -4.7, -5.4; IR (neat) $v_{\max } 2930,2857,1736,1328,1099$, $836 \mathrm{~cm}^{-1}$; CIMS ( $\mathrm{NH}_{3}$ gas): 600.7, 598.9, 408.5, 406.5, 359.5, 316.3, 222.3; HRMS (CI, $\left.\mathrm{NH}_{3}\right)$ $\mathrm{m} / \mathrm{z}$ calcd for $\mathrm{C}_{43} \mathrm{H}_{68} \mathrm{NO}_{6} \mathrm{SSi}_{2}[\mathrm{M}+\mathrm{H}]^{+}$782.431, found: 782.427 . 


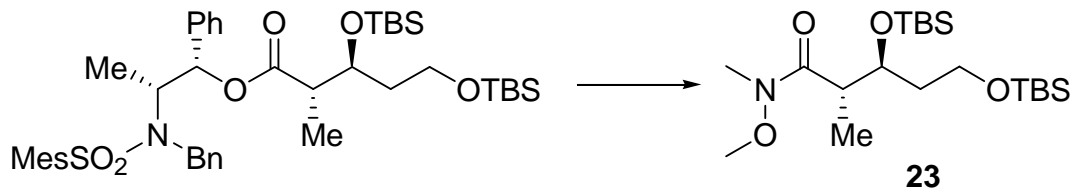

(2S,3S)-3,5-Bis-(tert-butyl-dimethylsilyloxy)-2-methyl-pentanoic acid methoxy-methylamide 23. To a slurry of [1(1S,2R),2S,3S]-3,5-Bis-(tert-butyl-dimethylsilyloxy)-2-methylpentanoic acid 2-( $N$-benzyl- $N$-mesitylenesulfonyl)amino]-1-phenyl-propyl ester (10.1 g, 12.9 mmol) and N,O-dimethylhydroxylamine hydrochloride (12.85 g, $131.7 \mathrm{mmol}$ ) in THF (90 mL) was added isopropylmagnesium chloride (2M solution in THF, $130 \mathrm{~mL}, 260 \mathrm{mmol}$ ) at $-20{ }^{\circ} \mathrm{C}$ over 75 minutes and under vigorous stirring. The reaction mixture was then stirred at $-20{ }^{\circ} \mathrm{C}$ for 1 hour and warmed to $5{ }^{\circ} \mathrm{C}$ over 1 hour. The cooling bath was then removed and the mixture was allowed to warm to rt where the slurry turned into an homogeneous brown solution which was then poured into a mixture of saturated $\mathrm{NH}_{4} \mathrm{Cl}$ solution, ice and ether. The organic layer was separated and the aqueous layer was extracted with ether. The combined organic extracts were washed with $1 \mathrm{M} \mathrm{HCl}$, saturated solution of $\mathrm{NaHCO}_{3}$ and brine, dried over $\mathrm{MgSO}_{4}$, filtered and concentrated. The crude residue was dissolved in a mixture of ether and hexanes (1:10, $50 \mathrm{~mL})$ and refrigerated overnight. Filtration of the crystals afforded (1S,2R)- $N$-Benzyl- $N$ mesitylenesulfonylnorephedrine $(4.7 \mathrm{~g}, 11.1 \mathrm{mmol}, 86 \%)$. Concentration of the residual liquid and flash chromatography (hexanes/AcOEt: 85/15) yielded a colorless oil (4.7 g, 11.2 mmol, 87 \%). $[\alpha]_{\mathrm{D}}^{20}+22.2$ (c 1.0, $\left.\mathrm{CHCl}_{3}\right) ;{ }^{1} \mathrm{H} \mathrm{NMR}\left(400 \mathrm{MHz}, \mathrm{CDCl}_{3}\right): \delta 4.09-4.13(\mathrm{~m}, 1 \mathrm{H}), 3.75$ (A of $\left.\mathrm{ABX}_{2}, J=6.2,10.0 \mathrm{~Hz}, 1 \mathrm{H}\right), 3.68$ (s, 3H), 3.64 (B of $\mathrm{ABX}_{2}, J=6.2,10.0 \mathrm{~Hz}, 1 \mathrm{H}$ ), 3.10-3.18 (obsc. M, 1H), 3.14 (s, 3H), 1.61-1.73 (m, 2H), 1.04 (d, J = 7.2 Hz, 3H), 0.86 (s, 9H), 0.84 (s, 9H), 0.04 (s, 3H), 0.02 (s, 6H), 0.00 (s, 3H); ${ }^{13} \mathrm{C}$ NMR (75 MHz, $\mathrm{CDCl}_{3}$ ): $\delta 176.1,70.5,61.3$, 58.9, 41.3, 35.8, 31.8, 25.9, 25.8, 18.1, 18.0, 12.4, -4.9, -5.4; IR (neat) $v_{\max } 2956,2857,1667$, 1255, 1098, $836 \mathrm{~cm}^{-1}$; CIMS (NH3 gas): 420.5, 404.5, 362.4, 359.4, 316.2, 171.1, 147.1, 73.1; HRMS (CI, $\mathrm{NH}_{3}$ ) m/z calcd for $\mathrm{C}_{20} \mathrm{H}_{46} \mathrm{NO}_{4} \mathrm{Si}_{2}[\mathrm{M}+\mathrm{H}]^{+}$420.297, found: 420.296. 


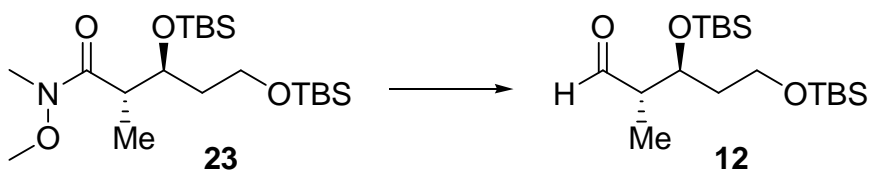

(2S,3S)-3,5-Bis-(tert-butyl-dimethylsilyloxy)-2-methyl-pentanal 12. To a solution of amide 23 (4.5 g, $10.7 \mathrm{mmol})$ in THF (100 mL) was slowly added DibalH (1M solution in hexanes, $15 \mathrm{~mL}$, $15 \mathrm{mmol}$ ) at $-78{ }^{\circ} \mathrm{C}$. The reaction mixture was then stirred at this temperature for 3 hours and carefully quenched with $960 \mu \mathrm{L}$ of $\mathrm{MeOH}$. The cooling bath was then removed and the mixture was allowed to warm to rt and poured into a mixture of saturated sodium potassium tartrate solution $(120 \mathrm{~mL})$ and ether $(180 \mathrm{~mL})$. After stirring at rt for 30 minutes, the organic layer was separated and the aqueous layer was extracted with ether. The combined organic extracts were washed with saturated sodium potassium tartrate solution, saturated solution of $\mathrm{NaHCO}_{3}$ and brine, dried over $\mathrm{MgSO}_{4}$, filtered and concentrated to give 12 as a colorless oil (3.9 g) which was used without further purifications. An analytical sample was obtained by filtration on a short silica plug neutralized with triethylamine (AcOEt used for elution). $[\alpha]_{\mathrm{D}}^{20}+13.3\left(c \mathrm{0.60}, \mathrm{CHCl}_{3}\right.$ ); ${ }^{1} \mathrm{H}$ NMR (400 MHz, $\left.\mathrm{CDCl}_{3}\right): \delta 9.71(\mathrm{~d}, J=2.0 \mathrm{~Hz}, 1 \mathrm{H}), 4.13$ (q, $\left.J=5.0 \mathrm{~Hz}, 1 \mathrm{H}\right), 3.67(\mathrm{t}, J=5.0$ Hz, 2H), 2.51-2.57 (m, 1H), 1.70-1.77 (m, 1H), 1.60-1.67 (m, 1H), 1.08 (d, $J=6.8 \mathrm{~Hz}, 3 \mathrm{H})$, 0.86 (s, 9H), 0.85 (s, 9H), 0.05 (s, 3H), 0.04 (s, 3H), 0.02 (s, 6H); ${ }^{13} \mathrm{C}$ NMR (75 MHz, CDCl $): \delta$ 205.2, 70.5, 59.2, 51.6, 37.7, 25.9, 25.8, 18.2, 18.1, 10.2, -4.4, -4.8, -5.4; IR (neat) $v_{\max } 2955$, 2930, 2858, 1728, 1256, 1101, $837 \mathrm{~cm}^{-1}$; CIMS (NH gas): 360.2, 359.2, 303.1, 229.1, 211.1, 171.1, 145.0, 73.0; HRMS (CI, $\mathrm{NH}_{3}$ ) m/z calcd for $\mathrm{C}_{18} \mathrm{H}_{40} \mathrm{O}_{3} \mathrm{Si}_{2}[\mathrm{M}]^{+} 360.252$, found: 360.246.

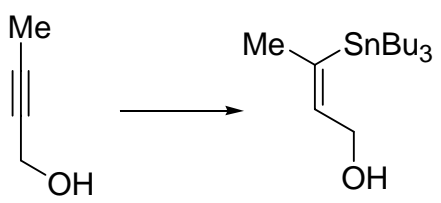

(Z)-3-Tributylstannanyl-but-2-en-1-ol. To a solution of 2-butyn-1-ol (10 mL, $134 \mathrm{mmol})$ in THF (250 mL) was added Red-Al ${ }^{\circledR}$ (65 wt. \% solution in toluene, $42 \mathrm{~mL}, 140 \mathrm{mmol}$ ) at $0{ }^{\circ} \mathrm{C}$ over 30 minutes. After the addition was complete, the resulting solution was stirred at rt for an additional 2 hours and tributyltin chloride (72 mL, $267 \mathrm{mmol})$ was added. The mixture was 
stirred at rt for 4 hours, quenched with water, and filtrated over a plug of Celite ${ }^{\circledR}$. The organic layer was separated and the aqueous layer was extracted with AcOEt. The combined organic extracts were washed brine, dried over $\mathrm{MgSO}_{4}$, filtered and concentrated. The crude residue was purified by flash chromatography over silica gel (gradient from hexanes to hexanes/AcOEt: 9/1) to give (2Z)-3-Tributylstannanyl-but-2-en-1-ol (43 g, $111 \mathrm{mmol}, 89 \%$ ) as a colorless oil. ${ }^{1} \mathrm{H}$ NMR (400 MHz, $\left.\mathrm{CDCl}_{3}\right): \delta 6.26$ (tq, $\left.J=1.6,6.8 \mathrm{~Hz},{ }^{3} J_{\mathrm{Sn}-\mathrm{H}, \text { trans }}=123 \mathrm{~Hz}, 1 \mathrm{H}\right), 4.01(\mathrm{t}, J=6.8$ $\mathrm{Hz}, 2 \mathrm{H}), 1.96$ (d, $\left.J=1.6 \mathrm{~Hz},{ }^{3} J_{\mathrm{Sn}-\mathrm{H}}=41 \mathrm{~Hz}, 3 \mathrm{H}\right), 1.23-1.43(\mathrm{~m}, 12 \mathrm{H}), 1.09$ (t, $\left.J=6.8 \mathrm{~Hz}, 1 \mathrm{H}\right)$, 0.88-1.01 (m, 15H); ${ }^{13} \mathrm{C}$ NMR (75 $\left.\mathrm{MHz} \mathrm{CDCl}_{3}\right): \delta 145.2,139.6,65.8,29.7,28.2,27.6,14.3$, 10.9; IR (neat) $v_{\max }$ 3610, 3012, 2958, 2930, 2853, 1464, 1378, 1069, $980 \mathrm{~cm}^{-1}$; CIMS $\left(\mathrm{NH}_{3}\right.$ gas): 363.2, 360.2, 344.2, 70.1; HRMS (CI, $\left.\mathrm{NH}_{3}\right) \mathrm{m} / \mathrm{z}$ calcd for $\mathrm{C}_{16} \mathrm{H}_{35} \mathrm{OSn}[\mathrm{M}+\mathrm{H}]^{+} 363.171$, found: 363.169 .

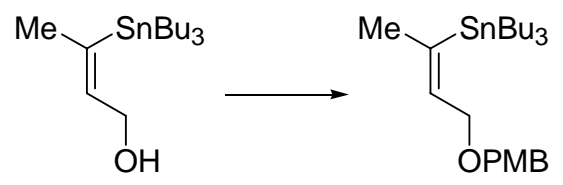

(Z)-Tributyl-[3-(4-methoxy-benzyloxy)-1-methyl-propenyl]-stannane. To a solution of (Z)-3Tributylstannanyl-but-2-en-1-ol (43 g, 119 mmol) in DMF (900 mL) was added sodium hydride (dry, $95 \%$, $3.3 \mathrm{~g}, 131 \mathrm{mmol}$ ) portionwise at $0{ }^{\circ} \mathrm{C}$. After the addition was complete, the resulting solution was stirred at rt for an additional 15 minutes and 4-methoxybenzyl chloride (23 mL, 170 mmol) was added. The mixture was stirred overnight at rt and carefully quenched with pH7 buffer $(500 \mathrm{~mL})$. The organic layer was separated and the aqueous layer was extracted with AcOEt. The combined organic extracts were washed brine, dried over $\mathrm{MgSO}_{4}$, filtered and concentrated. The crude residue was purified by flash chromatography over silica gel (hexanes/AcOEt: 95/5) to give (Z)-Tributyl-[3-(4-methoxy-benzyloxy)-1-methyl-propenyl]stannane XX (44 g, $91 \mathrm{mmol}, 76 \%$ ) as a colorless oil. ${ }^{1} \mathrm{H}$ NMR (400 MHz, $\left.\mathrm{CDCl}_{3}\right): \delta 7.25$ (d, $J$ $=8.4 \mathrm{~Hz}, 2 \mathrm{H}$ ), 6.85 (d, $J=8.4 \mathrm{~Hz}, 2 \mathrm{H}), 6.23$ (br. t, $\left.J=6.4 \mathrm{~Hz},{ }^{3} J_{\mathrm{Sn}-\mathrm{H}, \text { trans }}=128 \mathrm{~Hz}, 1 \mathrm{H}\right), 4.41$ (s, 2H), 3.85 (d, $J=6.4 \mathrm{~Hz}, 2 \mathrm{H}), 3.78$ (s, 3H), 1.93 (s, $\left.{ }^{3} J_{\mathrm{Sn}-\mathrm{H}}=42 \mathrm{~Hz}, 3 \mathrm{H}\right), 1.39-1.50$ (m, 6H), $1.24-$ 1.33 (m, 6H), 0.85-0.90 (m, 15H); ${ }^{13} \mathrm{C}$ NMR (75 MHz, $\mathrm{CDCl}_{3}$ ): $\delta$ 159.8, 144.3, 136.9, 130.2, 129.8, 113.9, 72.3, 72.1, 55.3, 29.2, 27.4, 27.1, 13.7, 10.1; IR (neat) $v_{\max } 2958,2929,1514,1361$ 
$\mathrm{cm}^{-1}$; CIMS (NH 3 gas): 467.0, 426.9, 425.0, 302.9, 291.0, 241.0, 175.0, 121.0; HRMS (CI, $\mathrm{NH}_{3}$ ) $\mathrm{m} / \mathrm{z}$ calcd for $\mathrm{C}_{24} \mathrm{H}_{42} \mathrm{O}_{2} \mathrm{Sn}[\mathrm{M}]^{+}$482.221, found: 482.216 .

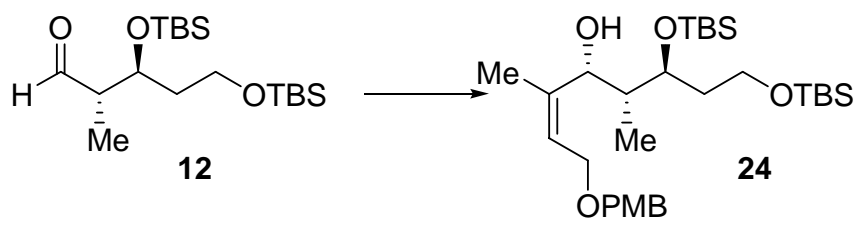

\section{(2Z,4R,5R,6S)-6,8-Bis-(tert-butyl-dimethylsilyloxy)-1-(4-methoxy-benzyloxy)-3,5-dimethyl-}

oct-2-en-4-ol 24. To a solution of (Z)-tributyl-[3-(4-methoxy-benzyloxy)-1-methyl-propenyl]stannane (6.85 g, $14.2 \mathrm{mmol})$ in THF (50 mL) was slowly added $n$-butyllithium (2.51M solution in hexanes, $5.55 \mathrm{~mL}, 13.9 \mathrm{mmol}$ ) at $-78{ }^{\circ} \mathrm{C}$. The resulting solution was stirred at $-78{ }^{\circ} \mathrm{C}$ for 30 minutes before adding the solution of aldehyde 12 (3.9 g, $10.7 \mathrm{mmol})$ in THF (50 mL) dropwise via cannula. The reaction mixture was stirred for an additional 2 hours and quenched with 100 $\mathrm{mL}$ of saturated $\mathrm{NH}_{4} \mathrm{Cl}$ solution. The organic layer was separated and the aqueous layer was extracted with ether. The combined organic extracts were washed brine, dried over $\mathrm{MgSO}_{4}$, filtered and concentrated. The crude residue was purified by flash chromatography over silica gel (gradient from hexanes to hexanes/AcOEt: 95/5) to give the alcohol 24 (4.3 g, $7.78 \mathrm{mmol}, 73$ \% over 2 steps) as a colorless oil. The de of this reaction was determined to be $6.5: 1$ by ${ }^{1} \mathrm{H}$ NMR analysis of the crude reaction mixture. $[\alpha]_{\mathrm{D}}^{20}+13.3$ (c $\left.0.60, \mathrm{CHCl}_{3}\right) ;{ }^{1} \mathrm{H} \mathrm{NMR}(400 \mathrm{MHz}$, $\left.\mathrm{CDCl}_{3}\right): \delta 7.25(\mathrm{~d}, J=8.4 \mathrm{~Hz}, 2 \mathrm{H}), 6.85(\mathrm{~d}, J=8.4 \mathrm{~Hz}, 2 \mathrm{H}), 5.45(\mathrm{t}, J=6.4 \mathrm{~Hz}, 1 \mathrm{H}), 4.56(\mathrm{~d}, J$ $=4.0 \mathrm{~Hz}, 1 \mathrm{H}), 4.42$ (s, 2H), 4.11 (A of $\mathrm{ABX}, J=8.0,12.0 \mathrm{~Hz}, 1 \mathrm{H}), 4.03$ (B of $\mathrm{ABX}, J=5.2$, $12.0 \mathrm{~Hz}, 1 \mathrm{H}), 3.85$ (td, J = 2.4, $6.0 \mathrm{~Hz}, 1 \mathrm{H}$ ), 3.77 (s, 3H), 3.53-3.63 (m, 2H), 3.27 (br. s, 1H), 1.76 (s, 3H), 1.62-1.77 (m, 3H), 1.01 (d, $J=7.2$ Hz, 3H), 0.89 (s, 9H), 0.88 (s, 9H), 0.08 (s, 6H), 0.03 (s, 3H), 0.01 (s, 3H); ${ }^{13} \mathrm{C}$ NMR (75 MHz, $\left.\mathrm{CDCl}_{3}\right): \delta$ 159.4, 140.9, 130.6, 129.6, 123.9, 113.9, 73.4, 71.9, 71.5, 65.7, 59.7, 55.2, 40.7, 37.0, 25.9, 25.8, 20.3, 18.1, 17.9, 11.4, -4.6, -4.7, 5.4, -5.5; IR (neat) $v_{\max }$ 3453, 2955, 2929, 2857, 1514, 1251, 1092, $836 \mathrm{~cm}^{-1}$; CIMS (NH3 gas): 403.4, 303.2, 283.2, 281.2, 171.1, 122.0, 121.0; HRMS (CI, $\mathrm{NH}_{3}$ ) m/z calcd for $\mathrm{C}_{30} \mathrm{H}_{57} \mathrm{O}_{5} \mathrm{Si}_{2}$ $[\mathrm{M}+\mathrm{H}]^{+}$553.374, found: 553.369 . 


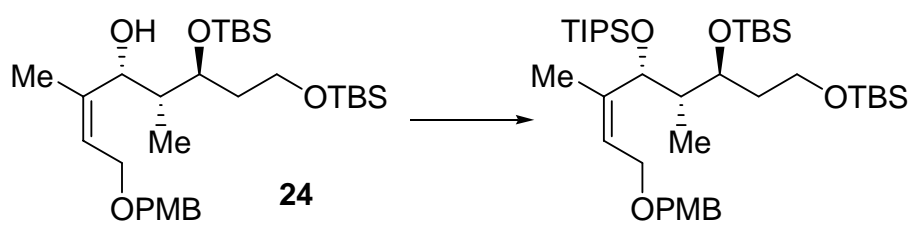

(2Z,4R,5S,6S)-6,8-Bis-(tert-butyl-dimethylsilyloxy)-1-(4-methoxy-benzyloxy)-3,5-dimethyl-

4-triisopropylsilyloxy-oct-2-ene. To a solution of alcohol 24 (4.3 g, $7.8 \mathrm{mmol}$ ) and 2,6-lutidine (1.8 $\mathrm{mL}, 15.4 \mathrm{mmol})$ in dichloromethane $(50 \mathrm{~mL})$ was added TIPSOTf (3.1 mL, $11.5 \mathrm{mmol})$ at 0 ${ }^{\circ} \mathrm{C}$. The resulting dark green solution was stirred at $0{ }^{\circ} \mathrm{C}$ for 2 hours and quenched with a saturated solution of $\mathrm{NaHCO}_{3}$. The organic layer was separated and the aqueous layer was extracted with dichloromethane. The combined organic extracts were washed with $1 \mathrm{M} \mathrm{HCl}$, saturated solution of $\mathrm{NaHCO}_{3}$ and brine, dried over $\mathrm{MgSO}_{4}$, filtered and concentrated. The crude residue was purified by flash chromatography over silica gel (hexanes/AcOEt: 95/5) to yield a colorless oil (5.4 g, $7.6 \mathrm{mmol}, 98 \%$ ). $[\alpha]_{\mathrm{D}}^{20}-6.0$ (c 0.50, $\left.\mathrm{CHCl}_{3}\right) ;{ }^{1} \mathrm{H} \mathrm{NMR}\left(400 \mathrm{MHz}, \mathrm{CDCl}_{3}\right): \delta$ 7.24 (d, $J=8.6 \mathrm{~Hz}, 2 \mathrm{H}), 6.85$ (d, $J=8.6 \mathrm{~Hz}, 2 \mathrm{H}), 5.42$ (t, $J=6.8 \mathrm{~Hz}, 1 \mathrm{H}), 4.45$ (A of ABq, $J=$ $11.6 \mathrm{~Hz}, 1 \mathrm{H}$ ), 4.36 (B of ABq, $J=11.6 \mathrm{~Hz}, 1 \mathrm{H}$ ), 4.18 (br. d, $J=8.0 \mathrm{~Hz}, 1 \mathrm{H}$ ), 4.08 (br. t, $J=9.8$ Hz, 1H), 3.70-3.95 (m, 1H), 3.79 (s, 3H), 3.67 (td, $J=4.0,9.2 \mathrm{~Hz}, 1 \mathrm{H}), 3.54$ (t, $J=6.8 \mathrm{~Hz}, 2 \mathrm{H})$, 1.82-1.89 (m, 1H), 1.75 (s, 3H), 1.50-1.58 (m, 1H), 1.24-1.29 (m, 1H), 0.99-1.50 (m, 24H), 0.88 (s, 9H), 0.86 (s, 9H), 0.02 (s, 6H), 0.01 (s, 3H),-0.01 (s, 3H); ${ }^{13} \mathrm{C}$ NMR (75 MHz, $\left.\mathrm{CDCl}_{3}\right): \delta$ 159.4, 139.4, 130.6, 129.6, 124.9, 114.0, 72.7, 68.7, 66.5, 61.1, 55.3, 44.3, 34.8, 26.1, 25.9, 18.4, 18.3, 18.2, 12.6, 10.7, -4.2, -4.7, -5.3; IR (neat) $v_{\max }$ 2958, 2930, 2855, 1510, 1101, $837 \mathrm{~cm}^{-1}$; CIMS ( $\mathrm{NH}_{3}$ gas): 571.3, 439.3, 303.1, 241.1, 171.0, 121.0; HRMS (CI, $\mathrm{NH}_{3}$ ) m/z calcd for $\mathrm{C}_{39} \mathrm{H}_{76} \mathrm{O}_{5} \mathrm{Si}_{3}[\mathrm{M}]^{+}$708.500, found: 708.499.

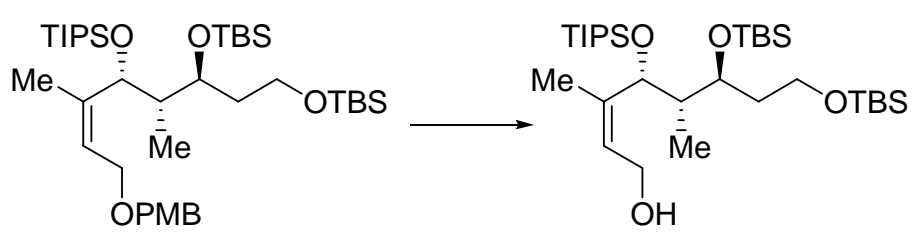

(2Z,4R,5S,6S)-6,8-Bis-(tert-butyl-dimethylsilyloxy)-3,5-dimethyl-4-triisopropylsilyloxy-oct-

2-en-1-ol. To a solution of (2Z,4R,5S,6S)-6,8-bis-(tert-butyl-dimethylsilyloxy)-1-(4-methoxybenzyloxy)-3,5-dimethyl-4-triisopropylsilyloxy-oct-2-ene (1.8 g, $2.54 \mathrm{mmol})$ in a mixture of 
dichloromethane $(60 \mathrm{~mL})$ and water $(6 \mathrm{~mL})$ was added 2,3-dichloro-5,6-dicyano-1,4benzoquinone $(620 \mathrm{mg}, 2.73 \mathrm{mmol})$ at $0{ }^{\circ} \mathrm{C}$. The resulting solution stirred at $0{ }^{\circ} \mathrm{C}$ for 90 minutes and diluted with water $(60 \mathrm{~mL})$. The organic layer was separated and the aqueous layer was extracted with dichloromethane. The combined organic extracts were washed with brine, dried over $\mathrm{MgSO}_{4}$, filtered and concentrated. The crude residue was purified by flash chromatography over silica gel (hexanes/AcOEt: 9/1) to yield a colorless oil (1.4 g, 2.38 mmol, 94 \%). [ $\alpha]_{D}^{20}$ 28.2 (c 0.80, $\mathrm{CHCl}_{3}$ ); ${ }^{1} \mathrm{H}$ NMR (400 MHz, $\mathrm{CDCl}_{3}$ ): $\delta 5.42$ (t, $\left.J=7.2 \mathrm{~Hz}, 1 \mathrm{H}\right), 4.31$ (d, $J=8.4$ Hz, 1H), 4.23 (t, $J=10.0$ Hz, 1H), 3.83-3.88 (m, 1H), 3.57-3.65 (m, 3H), 2.63 (br. s, 1H), 1.821.86 (m, 1H), 1.72 (s, 3H), 1.44-1.52 (m, 1H), 1.28-1.36 (m, 1H), 0.98-1.01 (m, 24H), 0.85 (s, 9H), 0.84 (s, 9H), 0.02 (s, 9H), -0.01 (s, 3H); ${ }^{13} \mathrm{C}$ NMR (75 MHz, $\mathrm{CDCl}_{3}$ ): $\delta 139.2,127.0,72.5$, 68.1, 60.4, 58.5, 43.5, 34.4, 26.2, 25.9, 18.5, 18.3, 18.2, 12.7, 10.7, -3.9, -4.7, -5.2, -5.4; IR (neat) $v_{\max }$ 3463, 2956, 2865, 1471, 1256, 1049, 1005, $836 \mathrm{~cm}^{-1}$; CIMS ( $\mathrm{NH}_{3}$ gas): 589.5, 571.5, 439.3, 303.2, 257.2, 215.1, 171.1, 133.1, 73.0; HRMS (CI, $\mathrm{NH}_{3}$ ) m/z calcd for $\mathrm{C}_{31} \mathrm{H}_{69} \mathrm{O}_{4} \mathrm{Si}_{3}[\mathrm{M}+\mathrm{H}]^{+}$ 589.450, found: 589.451 .

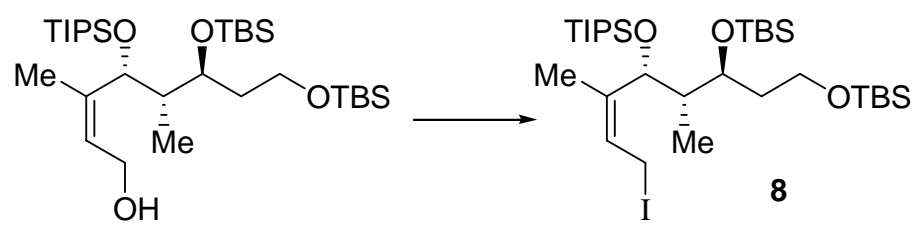

\section{(2Z,4R,5S,6S)-6,8-Bis-(tert-butyl-dimethylsilyloxy)-1-iodo-3,5-dimethyl-4-}

triisopropylsilyloxy-oct-2-ene 8. To a solution of triphenylphosphine (735 mg, $2.80 \mathrm{mmol}$ ) and imidazole (520 mg, $7.64 \mathrm{mmol})$ in dichloromethane $(15 \mathrm{~mL})$ was added iodine $(710 \mathrm{mg}, 2.80$ mmol) in one portion at $0{ }^{\circ} \mathrm{C}$. The mixture was then stirred for 10 minutes at $0{ }^{\circ} \mathrm{C}$ and to the resulting white slurry was added (2Z,4R,5S,6S)-6,8-bis-(tert-butyl-dimethylsilyloxy)-3,5dimethyl-4-triisopropylsilyloxy-oct-2-en-1-ol (1.50 g, $2.55 \mathrm{mmol})$ in dichloromethane (15 mL) via cannula. The colorless and homogeneous solution was stirred for 40 minutes at $0{ }^{\circ} \mathrm{C}$, concentrated and purified by flash chromatography over silica gel (gradient from hexanes to hexanes/AcOEt: 98/2) to give the iodide 8 (1.57 g, $2.25 \mathrm{mmol}, 88 \%)$ as a pale yellow oil. [ $\alpha]_{\mathrm{D}}^{20}$ 28.2 (c 0.80, $\mathrm{CHCl}_{3}$ ); ${ }^{1} \mathrm{H}$ NMR (400 MHz, $\mathrm{CDCl}_{3}$ ): $\delta 5.56$ (t, $\left.J=7.6 \mathrm{~Hz}, 1 \mathrm{H}\right), 4.31$ (br. s, $\left.1 \mathrm{H}\right)$, 3.82-3.99 (m, 2H), 3.53-3.69 (m, 3H), 1.82-1.86 (m, 1H), 1.75 (s, 3H), 1.53-1.60 (m, 1H), 1.29- 
1.38 (m, 1H), 1.01-1.07 (m, 24H), 0.87 (s, 9H), 0.85 (s, 9H), 0.02 (s, 6H), 0.01 (s, 3H), -0.01 (s, 3H); ${ }^{13} \mathrm{C}$ NMR (75 MHz, $\left.\mathrm{CDCl}_{3}\right): \delta 140.7,125.1,71.1,68.5,60.8,44.5,35.1,26.1,25.9$, 18.4, 18.2, 15.3, 10.8, -4.2, -4.6, -5.2, -5.3; IR (neat) $v_{\max }$ 2929, 2865, 1471, 1255, 1091, 1048, 836 $\mathrm{cm}^{-1}$; CIMS ( $\mathrm{NH}_{3}$ gas): 699.0, 655.3, 571.5, 467.1, 439.3, 367.1, 303.2, 265.2, 241.2, 197.1, 133.1; HRMS (CI, $\mathrm{NH}_{3}$ ) m/z calcd for $\mathrm{C}_{31} \mathrm{H}_{67} \mathrm{IO}_{3} \mathrm{Si}_{3}[\mathrm{M}]^{+}$698.344, found: 698.351.

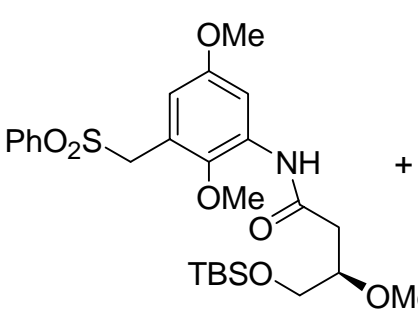

7

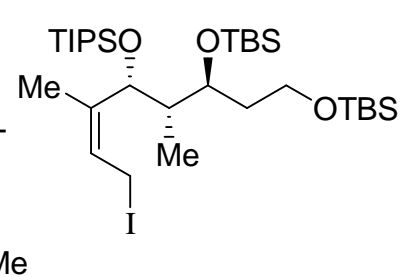

8

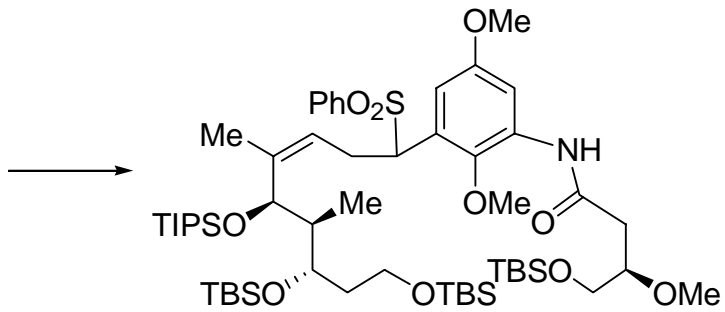

[1(1RS,3Z,5R,6S,7S),3R]-N-\{3-[1-Benzenesulfonyl-7,9-bis-(tert-butyl-dimethylsilyloxy)-4,6dimethyl-5-triisopropylsilyloxy-non-3-enyl]-2,5-dimethoxy-phenyl\}-4-(tert-butyldimethylsilyloxy)-3-methoxy-butyramide. A solution of sulfone 7 (3.9 g, $7.26 \mathrm{mmol}$ ) in THF (50 mL) was treated dropwise with a freshly prepared solution of LiHMDS (0.5M in THF, 32.0 $\mathrm{mL}, 16.0 \mathrm{mmol})$ at $-78{ }^{\circ} \mathrm{C}$. The resulting deep yellow solution was stirred at this temperature for 30 minutes and a solution of freshly prepared iodide 8 (3.3 g, $4.72 \mathrm{mmol})$ in THF (25 mL) was added via cannula. The reaction mixture was then stirred at $-78{ }^{\circ} \mathrm{C}$ for 2 hours, warmed to $-50{ }^{\circ} \mathrm{C}$ over 1 hour, stirred at $-50{ }^{\circ} \mathrm{C}$ for 3 hours and finally warmed to $-30{ }^{\circ} \mathrm{C}$ over 2 hours. The solution was then quickly poured into a mixture of $\mathrm{pH} 7$ buffer, ice and ether and stirred at rt. The organic layer was separated and the aqueous layer was extracted with AcOEt. The combined organic extracts were washed with brine, dried over $\mathrm{MgSO}_{4}$, filtered and concentrated. The crude residue was purified by flash chromatography over silica gel (gradient from hexanes/AcOEt: 9/1 to 7/3) to give the coupling product as an approximate 0.6/1 mixture of diastereoisomers and as a colorless oil (3.8 g, $3.43 \mathrm{mmol}, 73$ \%). ${ }^{1} \mathrm{H}$ NMR (400 $\left.\mathrm{MHz}, \mathrm{CDCl}_{3}\right): \delta 8.54,8.47$ (diastereoisomers, s, s, 1H), 8.02, 8.00 (diastereoisomers, d, $J=2.4 \mathrm{~Hz}, \mathrm{~d}, J=3.2 \mathrm{~Hz}, 1 \mathrm{H}$ ), 7.74, 7.69 (diastereoisomers, d, $J=7.6 \mathrm{~Hz}, \mathrm{~d}, J=7.6 \mathrm{~Hz}, 2 \mathrm{H}$ ), 7.55 (t, $J=6.8 \mathrm{~Hz}, 1 \mathrm{H}), 7.44,7.43$ (diastereoisomers, d, $J=7.6 \mathrm{~Hz}, \mathrm{~d}, J=8.0 \mathrm{~Hz}, 2 \mathrm{H}$ ), 6.78, 6.61 (diastereoisomers, s, s, $1 \mathrm{H}$ ), 4.82, 4.79 (diastereoisomers, t, $J=7.1 \mathrm{~Hz}, \mathrm{t}, J=6.9 \mathrm{~Hz}, 1 \mathrm{H}$ ), 4.69 (d, $J=10.8 \mathrm{~Hz}, 1 \mathrm{H}$ ), 4.44, 4.41 
(diastereoisomers, br. s, br. s, 1H), 4.18-4.23 (m, 1H), 3.77 (s, 3H), 3.63-3.76 (complex series of m, 4H), 3.42-3.60 (obsc. m, 4H), 3.52, 3.48 (diastereoisomers, s, s, 3H), 3.47, 3.43 (diastereoisomers, s, s, 3H), 2.62 (A of ABX, $J=2.8,15.6 \mathrm{~Hz}, 1 \mathrm{H}$ ), 2.52 (B of ABX, $J=8.0$, 15.6 Hz, 1H), 1.78-1.83 (br. m, 2H), 1.57 (s, 3H), 1.30-1.38 (br. m, 2H), 0.85-1.06 (complex series of m, $51 \mathrm{H}$ ), -0.04-0.09 (complex series of s, $18 \mathrm{H}$ ); ${ }^{13} \mathrm{C}$ NMR $\left(75 \mathrm{MHz}, \mathrm{CDCl}_{3}\right.$ ): $\delta 168.7$, 156.2, 140.5, 137.3, 135.0, 134.2, 133.7, 133.2, 132.4, 130.9, 129.7, 128.3, 127.6, 110.3, 102.6, 78.9, 71.7, 71.5, 61.0, 56.9, 55.6, 45.5, 45.1, 36.7, 31.3, 29.8, 29.4, 25.8, 18.4, 18.3, 12.8, 12.7, 10.1, -4.3, -4.4; IR (neat) $v_{\max }$ 3430, 3340, 2950, 2862, 1684, $1424 \mathrm{~cm}^{-1}$; CIMS ( $\mathrm{NH}_{3}$ gas): 1108.6, 1045.4, 965.0, 908.3, 793.5, 661.6, 605.0, 528.5, 420.2, 303.2, 231.2, 115.1; HRMS (CI, $\mathrm{NH}_{3}$ ) $\mathrm{m} / \mathrm{z}$ calcd for $\mathrm{C}_{57} \mathrm{H}_{106} \mathrm{NO}_{10} \mathrm{SSi}_{4}[\mathrm{M}+\mathrm{H}]^{+}$1108.661, found: 1108.657.
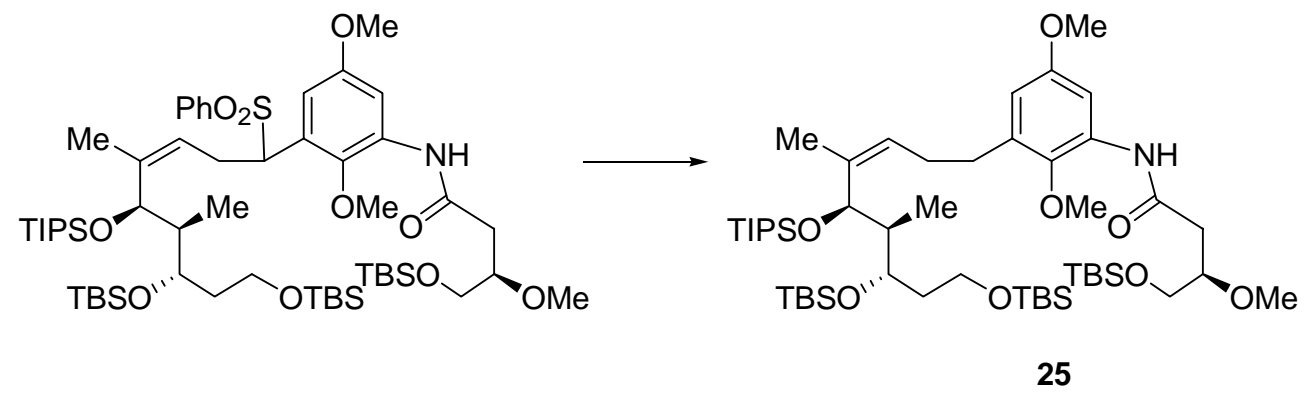

\section{[1(3Z,5R,6S,7S),3R]-N-\{3-[7,9-Bis-(tert-butyl-dimethylsilyloxy)-4,6-dimethyl-5-}

\section{triisopropylsilyloxy-non-3-enyl]-2,5-dimethoxy-phenyl\}-4-(tert-butyl-dimethylsilyloxy)-3-}

methoxy-butyramide 25. To a solution of the above diastereoisomeric sulfones (2.5 g, 2.25 $\mathrm{mmol})$ in $\mathrm{MeOH}(40 \mathrm{~mL})$ was added anhydrous sodium hydrogenphosphate (1.3 g, $9.23 \mathrm{mmol})$ at $-20{ }^{\circ} \mathrm{C}$. The resulting mixture was stirred at this temperature for 10 minutes, treated with sodium mercury amalgam (5\% Na, 11.25 g) and kept under vigorous stirring at $-20{ }^{\circ} \mathrm{C}$ for 90 minutes. The crude reaction mixture was filtrated over a short plug of silica gel (rinsed several times with AcOEt), concentrated and dissolved in AcOEt and water. The organic layer was separated and the aqueous layer was extracted with AcOEt. The combined organic extracts were washed with brine, dried over $\mathrm{MgSO}_{4}$, filtered and concentrated. The crude residue was purified by flash chromatography over silica gel (hexanes/AcOEt: 9/1) to give the desulfonylated product 25 as a colorless oil (2.1 g, $2.17 \mathrm{mmol}, 96 \%)$. [ $\alpha]_{\mathrm{D}}^{20}-18.4\left(c\right.$ 0.62, $\left.\mathrm{CHCl}_{3}\right) ;{ }^{1} \mathrm{H} \mathrm{NMR}(400 \mathrm{MHz}$, 
$\left.\mathrm{CDCl}_{3}\right): \delta 8.72(\mathrm{~s}, 1 \mathrm{H}), 7.90(\mathrm{~d}, J=2.8 \mathrm{~Hz}, 1 \mathrm{H}), 6.39$ (d, $\left.J=2.8 \mathrm{~Hz}, 1 \mathrm{H}\right), 5.20(\mathrm{~d}, J=4.4 \mathrm{~Hz}$, 1H), 4.27 (d, $J=9.2 \mathrm{~Hz}, 1 \mathrm{H}$ ), 3.75 (s, 3H), 3.61-3.72 (obsc. m, 4H), 3.66 (s, 3H), 3.50-3.56 (obsc. m, 2H), 3.51 (s, 3H), 2.64 (A of ABq, $J=2.4,15.6 \mathrm{~Hz}, 1 \mathrm{H}), 2.59$ (t, $J=7.4 \mathrm{~Hz}, 2 \mathrm{H}$ ), 2.56 (B of ABq, $J=7.6,15.6 \mathrm{~Hz}, 1 \mathrm{H}$ ), 2.37-2.43 (m, 1H), 2.19 (br. s, 1H), 1,87 (br. s, 1H), 1.70 (s, 3H), 1.49-1.58 (m, 1H), 1.26-1.34 (m, 1H), 1.00-1.04 (m, 24 H), 0.88 (s, 9H), 0.85 (s, 9H), 0.82 (s, 9H), 0.06 (s, 3H), 0.05 (s, 3H), 0.01 (s, 3H), -0.02 (s, 3H), -0.05 (s, 6H); ${ }^{13} \mathrm{C}$ NMR (75 MHz, $\left.\mathrm{CDCl}_{3}\right): \delta 169.8,156.3,141.1,136.7,135.0,132.5,127.1,110.6,103.6,79.0,72.6,68.8,64.0$, 61.2, 61.1, 58.0, 55.6, 44.4, 40.4, 34.8, 30.1, 29.3, 26.0, 25.9, 18.4, 18.3, 12.7, 10.8, -4.2, -4.7, -

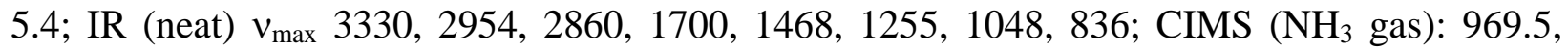
925.2, 911.4, 795.2, 662.4, 606.6, 530.3, 492.4, 422.3, 303.2, 231.1; HRMS (CI, $\mathrm{NH}_{3}$ ) m/z calcd for $\mathrm{C}_{51} \mathrm{H}_{102} \mathrm{NO}_{8} \mathrm{Si}_{4}[\mathrm{M}+\mathrm{H}]^{+}$968.668, found: 968.668 .

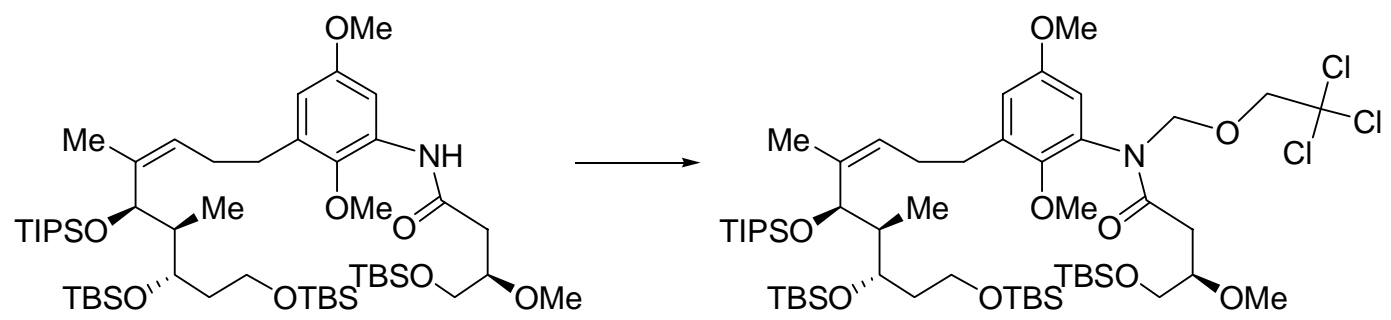

25

\section{[1(3Z,5R,6S,7S),3R]-N-\{3-[7,9-Bis-(tert-butyl-dimethylsilyloxy)-4,6-dimethyl-5-}

\section{triisopropylsilyloxy-non-3-enyl]-2,5-dimethoxy-phenyl\}-4-(tert-butyl-dimethylsilyloxy)-3-}

methoxy- $N$-(2,2,2-trichloro-ethoxymethyl)-butyramide. To a solution of amide 25 (540 mg, $0.557 \mathrm{mmol})$ in THF (14 mL) was added potassium hydride (washed with anhydrous hexanes, $224 \mathrm{mg}, 5.58 \mathrm{mmol}$ ) at $0{ }^{\circ} \mathrm{C}$. The resulting mixture was stirred at this temperature for 9 minutes and chloromethyl 2,2,2-trichloroethyl ether ${ }^{\mathrm{S} 7}$ (365 $\left.\mu \mathrm{L}, 2.79 \mathrm{mmol}\right)$ was added. The cooling bath was then removed and the reaction mixture was stirred at rt for 20 minutes, cooled to $0{ }^{\circ} \mathrm{C}$ and carefully quenched with a saturated solution of $\mathrm{NaHCO}_{3}$. The organic layer was separated and

S7 Chloromethyl 2,2,2-trichloroethyl ether was prepared by extension of the Boeckman protocol for benzyl chloromethyl ether: Connor, D. S.; Klein, G. W.; Taylor, G. N.; Boeckman, R. K., Jr.; Medwid, J. B. Organic Syntheses; Wiley: New York, 1988; Collect. Vol. VI, p 101. Also see: Salomaa, P.; Linnantie, R. Acta Chem. Scand. 1960, 14, 777. After distillation, this reagent was stored over anhydrous calcium chloride at $-4{ }^{\circ} \mathrm{C}$. ${ }^{1} \mathrm{H}$ NMR $(400$ $\left.\mathrm{MHz}, \mathrm{CDCl}_{3}\right): \delta 5.61(\mathrm{~s}, 2 \mathrm{H}), 4.23(\mathrm{~s}, 2 \mathrm{H})$. 
the aqueous layer was extracted with AcOEt. The combined organic extracts were washed with brine, dried over $\mathrm{MgSO}_{4}$, filtered and concentrated. The crude residue was purified by flash chromatography over silica gel (hexanes/AcOEt: 95/5) to give the protected amide as an approximate 0.7/1 mixture of atropoisomers and as a colorless oil (601 mg, $0.532 \mathrm{mmol}, 95 \%$ ). $[\alpha]_{\mathrm{D}}^{20}+1.1$ ( $c$ 1.31, $\left.\mathrm{CHCl}_{3}\right) ;{ }^{1} \mathrm{H} \mathrm{NMR}\left(400 \mathrm{MHz}, \mathrm{CDCl}_{3}\right): \delta 6.69,6.59$ (atropoisomers, d, $J=2.8$ Hz, d, $J=3.2 \mathrm{~Hz}, 1 \mathrm{H}$ ), 6.57, 6.49 (atropoisomers, d, $J=3.2 \mathrm{~Hz}$, d, $J=2.8 \mathrm{~Hz}, 1 \mathrm{H}$ ), 5.79, 5.77 (atropoisomers, A of ABq, $J=10.4 \mathrm{~Hz}, \mathrm{~A}$ of ABq, $J=10.4 \mathrm{~Hz}, 1 \mathrm{H}), 5.04-5.13(\mathrm{~m}, 1 \mathrm{H}), 4.74$, 4.70 (atropoisomers, B of ABq, $J=10.4 \mathrm{~Hz}, \mathrm{~B}$ of ABq, $J=10.4 \mathrm{~Hz}, 1 \mathrm{H}$ ), 4.16-4.29 (complex series of m, 3H), 3.61-3.71 (obsc. m, 1H), 3.60 (s, 3H), 3.37-3.56 (complex series of m, 5H), 3.52, 3.47 (atropoisomers, s, s, 3H), 3.29, 3.24 (atropoisomers, s, s, 3H), 2.51-2.61 (m, 1H), 2.382.47 (m, 1H), 2.18-2.36 (complex series of m, 3H), 2.06-2.19 (m, 1H), 1.75-1.82 (m, 3H), 1.61 (s, 1H), 1.12-1.23 (m, 2H), 0.90-0.94 (m, 24H), 0.74 (s, 9H), 0.73, 0.71 (atropoisomers, s, s, 9H), 0.70, 0.69 (atropoisomers, s, s, 9H), $-0.09,-0.12,-0.14,-0.16$ (s, s, s, s, $18 \mathrm{H}) ;{ }^{13} \mathrm{C}$ NMR (75 $\mathrm{MHz}_{\mathrm{CDCl}}$ ): $\delta 173.8,173.3,156.1,156.0,147.6,147.5,137.8,137.2,136.9,135.2,134.9$, 126.8, 116.2, 116.1, 113.0, 112.8, 97.3, 97.1, 82.5, 81.9, 79.4, 78.7, 78.5, 78.2, 76.4, 72.6, 68.7, 64.3, 64.0, 61.5, 61.3, 60.9, 58.5, 58.1, 55.6, 55.5, 44.3, 36.6, 36.3, 34.7, 30.2, 30.0, 29.3, 26.0, 25.9, 25.8, 18.4, 18.2, 12.7, 10.8, -4.1, -4.7, -5.4, -5.5; IR (neat) $v_{\max } 2930,2859,1743,1675$, 1471, 1252, 1090; CIMS ( $\mathrm{NH}_{3}$ gas): 1128.7, 1072.3, 911.2, 798.4, 584.0, 422.1, 351.9, 303.1, 231.1, 204.1, 89.0; HRMS (CI, $\mathrm{NH}_{3}$ ) $\mathrm{m} / \mathrm{z}$ calcd for $\mathrm{C}_{54} \mathrm{H}_{105} \mathrm{Cl}_{3} \mathrm{NO}_{9} \mathrm{Si}_{4}[\mathrm{M}+\mathrm{H}]^{+} 1128.593$, found: 1128.598 .

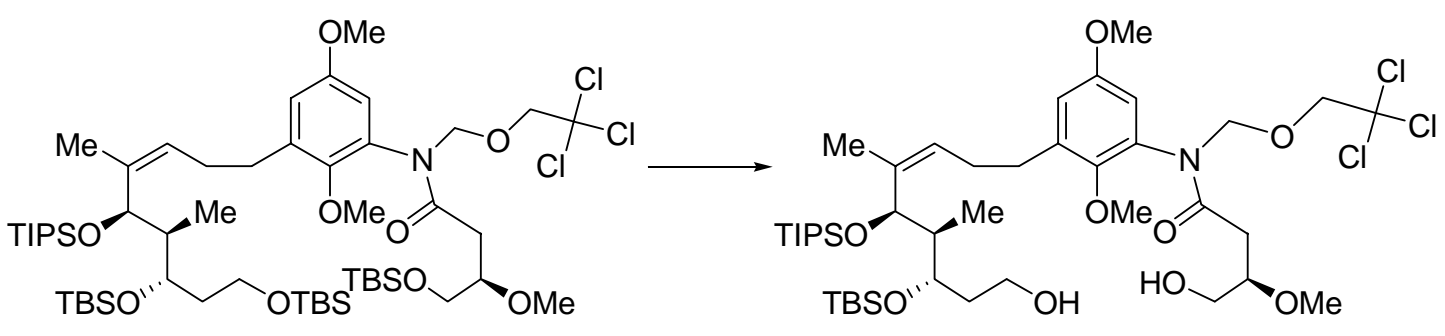

[1(3Z,5R,6S,7S),3R]-N-\{3-[7-(tert-butyl-dimethylsilyloxy)-9-hydroxy-4,6-dimethyl-5triisopropylsilyloxy-non-3-enyl]-2,5-dimethoxy-phenyl $\}-4-h y d r o x y-3-m e t h o x y-N-(2,2,2-$ trichloro-ethoxymethyl)-butyramide. In a $15 \mathrm{~mL}$ polyethylene vial, the above amide (601 mg, 
$0.532 \mathrm{mmol})$ was dissolved in THF $(5.5 \mathrm{~mL})$. The vial was cooled to $0{ }^{\circ} \mathrm{C}$ and hydrogen fluoride-pyridine complex buffered with pyridine $(3.2 \mathrm{~mL}$ of a stock solution made from $3.0 \mathrm{~mL}$ of pyridine, $7.5 \mathrm{~mL}$ of THF and $2.7 \mathrm{~mL}$ of hydrogen fluoride-pyridine) was added. The reaction mixture was stirred at $0{ }^{\circ} \mathrm{C}$ for 1 hour and at $\mathrm{rt}$ for another hour and carefully poured into a saturated $\mathrm{NaHCO}_{3}$ solution. The organic layer was separated and the aqueous layer was extracted with AcOEt. The combined organic extracts were dried over $\mathrm{MgSO}_{4}$, filtered and concentrated. The crude residue was purified by flash chromatography over silica gel (hexanes/AcOEt: 6/4) to give the deprotected diol as an approximate 0.8/1 mixture of atropoisomers and as a white foam (436 mg, 0.483 mmol, 91 \%). $[\alpha]_{\mathrm{D}}^{20}+10.3$ (c 0.32, $\mathrm{CHCl}_{3}$ ); ${ }^{1} \mathrm{H}$ NMR (400 MHz, $\left.\mathrm{CDCl}_{3}\right): \delta 6.78$, 6.69 (atropoisomers, br. s, br. s, 1H), 6.68, 6.67 (atropoisomers, br. s, br. s, 1H), 5.82, 5.78 (atropoisomers, A of ABq, $J=10.4 \mathrm{~Hz}, \mathrm{~A}$ of ABq, $J=11.2 \mathrm{~Hz}, 1 \mathrm{H}), 5.18-5.24(\mathrm{~m}, 1 \mathrm{H}), 4.87$, 4.86 (atropoisomers, B of ABq, $J=10.4 \mathrm{~Hz}$, B of ABq, $J=11.2 \mathrm{~Hz}, 1 \mathrm{H}$ ), 4.37 (A' of A'B'q, $J=$ $11.8 \mathrm{~Hz}, 1 \mathrm{H}$ ), 4.31, 4.30 (atropoisomers, B' of A’B'q, $J=11.8 \mathrm{~Hz}, \mathrm{~B}$ ' of A'B'q, $J=11.8 \mathrm{~Hz}$, 1H), 4.25 (d, $J=9.2 \mathrm{~Hz}, 1 \mathrm{H}), 3.76-3.81$ (m, 1H), 3.72 (s, 3H), 3.62-7.73 (obsc. m, 2H), 3.63, 3.61 (atropoisomers, s, s, 3H), 3.42-3.56 (complex series of m, 3H), 3.32 (s, 3H), 2.60-2.72 (m, 1H), 2.40-2.58 (complex series of m, 3H), 2.28-2.39 (complex series of m, 3H), 2.14 (br. s, 1H), 1.87 (br. s, 1H), 1.71 (s, 3H), 1.60-1.67 (m, 1H), 0.99-1.05 (m, 24H), 0.84 (s, 9H), 0.01 (s, 3H), 0.01 (s, 3H); ${ }^{13} \mathrm{C}$ NMR (75 MHz, $\left.\mathrm{CDCl}_{3}\right): \delta 173.4,173.2,156.3,156.2,147.4,147.3,137.7$, 137.4, 137.1, 135.3, 135.1, 126.7, 126.6, 116.3, 116.0, 112.4, 97.1, 97.0, 82.6, 82.3, 79.1, 78.8, 78.5, 78.2, 72.3, 70.1, 63.1, 62.6, 61.7, 61.5, 61.0, 60.9, 57.5, 55.6, 44.1, 35.7, 35.6, 34.1, 34.0, 30.7, 30.6, 29.7, 29.3, 25.8, 18.3, 18.2, 17.9, 12.7, 12.6, 10.5, 10.4, -4.2, -4.9; IR (neat) $v_{\max }$ 3400-3200 (br), 2930, 2857, $1670 \mathrm{~cm}^{-1}$; CIMS ( $\mathrm{NH}_{3}$ gas): 899.1, 897.2, 867.1, 829.2, 277.0, 165.1, 135.0, 89.0; HRMS (CI, $\mathrm{NH}_{3}$ ) $\mathrm{m} / \mathrm{z}$ calcd for $\mathrm{C}_{42} \mathrm{H}_{76} \mathrm{Cl}_{3} \mathrm{NO}_{9} \mathrm{Si}_{2}$ [M] 899.412, found: 899.415. 


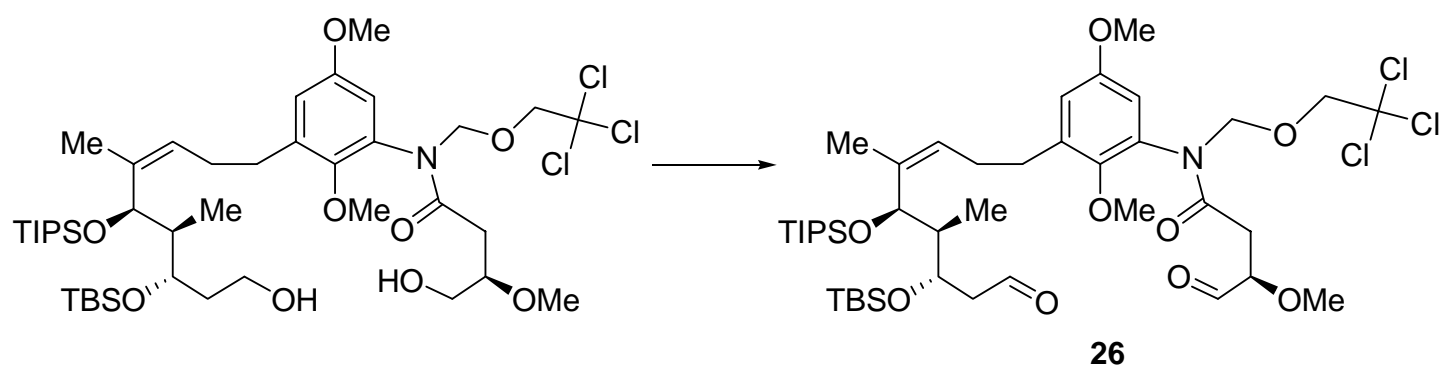

\section{[1(3Z,5R,6S,7S),3R]-N-\{3-[7-(tert-butyl-dimethylsilyloxy)-4,6-dimethyl-9-oxo-5-}

triisopropylsilyloxy-non-3-enyl]-2,5-dimethoxy-phenyl\}-3-methoxy-4-oxo- $N$-(2,2,2-

trichloro-ethoxymethyl)-butyramide 26. A solution of the above diol (400 $\mathrm{mg}, 0.444 \mathrm{mmol}$ ) in DMSO (9 mL) was treated with triethylamine $(3.2 \mathrm{~mL}, 22.7 \mathrm{mmol})$ and sulfur trioxide pyridine complex (1.08 g, $6.78 \mathrm{mmol})$. The resulting mixture was kept under vigorous stirring at rt for 80 minutes and diluted with water and AcOEt. The organic layer was separated and the aqueous layer was extracted with AcOEt. The combined organic extracts were washed with brine, dried over $\mathrm{MgSO}_{4}$, filtered and concentrated. The crude residue was purified by flash chromatography over silica gel (hexanes/AcOEt: 1/1) to give the bis-aldehyde 26 as an approximate 0.8/1 mixture of atropoisomers and as a white foam (361 mg, $0.402 \mathrm{mmol}, 91 \%)$. $[\alpha]_{\mathrm{D}}^{20}+14.7$ (c 1.03, $\mathrm{CHCl}_{3}$ ); ${ }^{1} \mathrm{H}$ NMR (400 MHz, $\mathrm{CDCl}_{3}$ ): $\delta$ 9.79, 9.73 (atropoisomers, s, s, 1H), 9.70 (br. s, 1H), 6.74, 6.72 (atropoisomers, s, s, 1H), 6.69 (s, 1H), 5.81, 5.79 (atropoisomers, A of ABq, $J=9.8 \mathrm{~Hz}, \mathrm{~A}$ of ABq, $J=10.2 \mathrm{~Hz}, 1 \mathrm{H}$ ), 5.24 (br. s, $1 \mathrm{H}$ ), 4.89, 4.86 (atropoisomers, B of ABq, $J=9.8 \mathrm{~Hz}$, B of ABq, $J=10.2 \mathrm{~Hz}, 1 \mathrm{H}$ ), 4.29-4.41 (complex series of m, 2H), 4.20 (d, $J=9.2 \mathrm{~Hz}, 1 \mathrm{H}$ ), 4.02-4.07 (m, 2H), 3.73 (s, 3H), 3.60 (s, 3H), 3.45, 3.44 (atropoisomers, s, s, 3H), 2.50-2.72 (complex series of m, 5H), 2.77-2.33 (m, 1H), 2.01-2.14 (m, 2H), 1.90-1.96 (m, 1H), 1.73 (s, 3H), 11.04 (br. s, 24H), 0.82 (s, 9H), 0.04 (s, 3H), -0.02 (s, 3H); ${ }^{13} \mathrm{C}$ NMR (75 MHz, $\mathrm{CDCl}_{3}$ ): $\delta$ 203.2, 203.0, 202.7, 171.8, 171.7, 156.3, 147.5, 147.4, 137.5, 137.4, 136.9, 135.0, 134.9, 127.0, 116.2, 116.1, 112.5, 112.3, 97.1, 82.6, 82.4, 82.3, 79.0, 78.9, 72.3, 67.9, 61.7, 61.6, 59.0, 58.8, 55.7, 46.3, 44.2, 35.8, 35.6, 30.2, 29.7, 29.4, 29.3, 25.7, 18.3, 18.2, 12.7, 10.7, -4.3, -4.8; IR (neat) $v_{\max }$ 3400-3200 (br), 3340, 1730, 1719, 1668, $1580 \mathrm{~cm}^{-1}$; CIMS ( $\mathrm{NH}_{3}$ gas): 898.8, 897.2, 748.7, 682.2, 532.3, 468.0, 270.1, 246.0, 204.1, 124.1; HRMS (CI, $\mathrm{NH}_{3}$ ) m/z calcd for $\mathrm{C}_{42} \mathrm{H}_{73} \mathrm{Cl}_{3} \mathrm{NO}_{9} \mathrm{Si}_{2}$ $[\mathrm{M}+\mathrm{H}]^{+}$896.389, found: 896.379. 


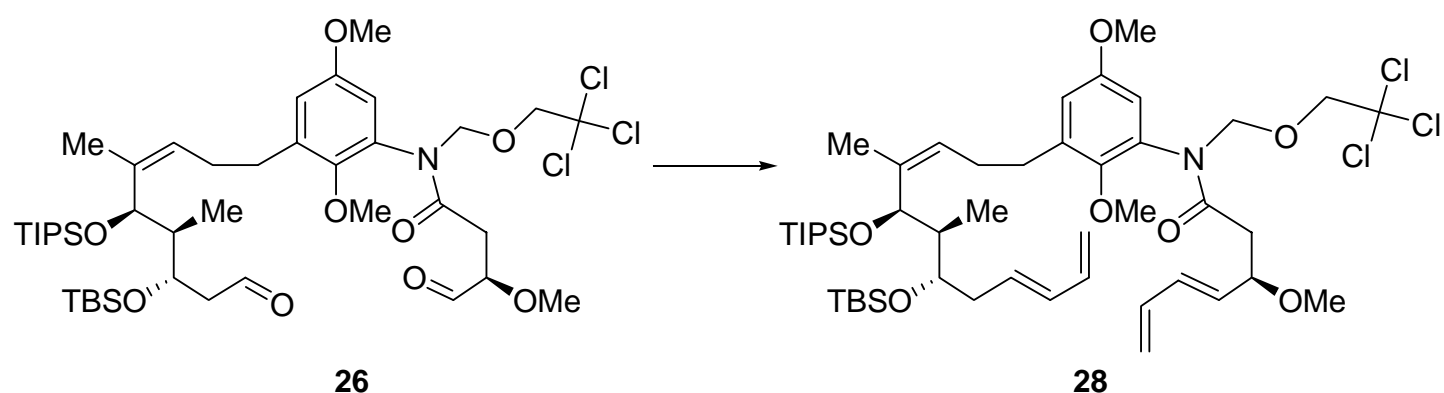

[1(3Z,5R,6S,7S,9E),3R,4E]-3-Methoxy-hepta-4,6-dienoic

acid \{3-[7-(tert-butyldimethylsilyloxy)-4,6-dimethyl-5-triisopropylsilyloxy-dodeca-3,9,11-trienyl]-2,5-dimethoxyphenyl\}-(2,2,2-trichloro-ethoxymethyl)-amide 28.

PROCEDURE A (Yamamoto's allylation / Peterson olefination): To a solution of bisaldehyde 26 (61 mg, $0.068 \mathrm{mmol}$ ) and (E)-trimethyl-(3-tributylstannanyl-propenyl)-silane $27^{\mathrm{S} 8}$ (110 mg, $0.273 \mathrm{mmol})$ in dichloromethane $(1 \mathrm{~mL})$ was added boron trifluoride diethyletherate $(26 \mu \mathrm{L}, 0.205 \mathrm{mmol})$ at $-78{ }^{\circ} \mathrm{C}$. The resulting mixture was stirred for 36 hours at $-78{ }^{\circ} \mathrm{C}, 3$ hours at $-50{ }^{\circ} \mathrm{C}$ and quenched with a saturated solution of $\mathrm{NaHCO}_{3}$. The organic layer was separated and the aqueous layer was extracted with dichloromethane. The combined organic extracts were washed with brine, dried over $\mathrm{MgSO}_{4}$, filtered and concentrated. Tin residues were removed by reverse phase chromatography, eluting first with acetonitrile to remove those residues and with a fast gradient of acetonitrile/dichloromethane (from 9/1 to 1/9). Bis-allylation product was so obtained as a complex mixture of diastereoisomers and atropoisomers. This material was used without further purification.

This complex mixture was dissolved in THF $(1 \mathrm{~mL})$ and treated with potassium tertbutoxide (76 mg, $0.68 \mathrm{mmol}$ ) for 2 hours and saturated $\mathrm{NH}_{4} \mathrm{Cl}$ solution was then added. The organic layer was separated and the aqueous layer was extracted with AcOEt. The combined organic extracts were washed with brine, dried over $\mathrm{MgSO}_{4}$, filtered and concentrated. The crude residue was purified by flash chromatography over silica gel (hexanes/AcOEt: 95/5) to give the desired bis-diene 28 as an approximate 0.7/1 mixture of atropoisomers and as a white foam (39 mg, $0.041 \mathrm{mmol}, 61 \%$ over the 2 steps sequence).

PROCEDURE B (Wittig / reduction / Swern oxidation / Wittig): A solution of bis-aldehyde 26 (201 mg, $0.224 \mathrm{mmol})$ in dichloromethane $(25 \mathrm{~mL}$ ) was treated with (carbethoxymethylene)-

\footnotetext{
${ }^{\text {s8 }}$ Yamamoto, Y.; Saito, Y.; Maruyama, K. J. Organomet. Chem. 1985, 292, 311-318.
} 
triphenylphosphorane (690 mg, $1.98 \mathrm{mmol}$ ). After 20 hours at rt, the crude reaction mixture was concentrated and filtrated over a short plug of silica gel (hexanes/AcOEt: 7:3) providing the corresponding bis-conjugated ester.

This ester was dissolved in THF $(20 \mathrm{~mL})$ and cooled to $-78{ }^{\circ} \mathrm{C}$. Diisobutylaluminium hydride (1M solution in hexanes, $1.0 \mathrm{~mL}, 1.0 \mathrm{mmol})$ was then added and the reaction mixture was stirred 1 hour at $-78{ }^{\circ} \mathrm{C}$, warmed to rt over 1 hour and quenched with $15 \mathrm{~mL}$ of saturated potassium sodium tartrate. The mixture was stirred vigorously until it turned clear. The organic layer was separated and the aqueous layer was extracted with AcOEt. The combined organic extracts were washed with brine, dried over $\mathrm{MgSO}_{4}$, filtered and concentrated to give the corresponding bis-allylic alcohol used without further purification in the next step.

DMSO (175 $\mu \mathrm{L}, 2.47 \mathrm{mmol}$ ) was added to a solution of oxalyl chloride (105 $\mu \mathrm{L}, 1.20$ $\mathrm{mmol})$ in dichloromethane $(6.5 \mathrm{~mL})$ at $-78{ }^{\circ} \mathrm{C}$. The resulting solution was stirred at $-78{ }^{\circ} \mathrm{C}$ for 30 minutes and a solution of the above bis-allylic alcohol in dichloromethane $(4 \mathrm{~mL})$ was added dropwise via cannula. The reaction mixture was stirred for 55 minutes at $-78{ }^{\circ} \mathrm{C}$, triethylamine (470 $\mu \mathrm{L}, 3.37 \mathrm{mmol}$ ) was added and the mixture was warmed to rt over 3 hours and concentrated. The crude residue was purified by filtration over a short plug of silica gel (hexanes/AcOEt: 7/3) to give the corresponding bis-aldehyde used without further purification in the next step.

To a suspension of methyltriphenylphosphonium bromide (553 mg, $1.55 \mathrm{mmol}$ ) in THF (18.5 mL) at $-78{ }^{\circ} \mathrm{C}$ was added $n$-butyllithium (2.51M in hexanes, $\left.495 \mu \mathrm{L}, 1.24 \mathrm{mmol}\right)$. The resulting yellow solution was stirred at $-78{ }^{\circ} \mathrm{C}$ for 40 minutes and a solution of the above bisaldehyde in THF (7 mL) was added via cannula. The reaction mixture was warmed to $0{ }^{\circ} \mathrm{C}$ over 1 hour and quenched with $10 \mathrm{~mL}$ of saturated $\mathrm{NH}_{4} \mathrm{Cl}$ solution. The organic layer was separated and the aqueous layer was extracted with AcOEt. The combined organic extracts were washed with brine, dried over $\mathrm{MgSO}_{4}$, filtered and concentrated. The crude residue was purified by flash chromatography over silica gel (hexanes/AcOEt: 95/5) to give the desired bis-diene $\mathbf{2 8}$ as an approximate 0.7/1 mixture of atropoisomers and as a white foam (137 mg, $0.145 \mathrm{mmol}, 65 \%$ over the 4 steps sequence).

$[\alpha]_{\mathrm{D}}^{20}-12.7\left(c\right.$ 0.71, $\left.\mathrm{CHCl}_{3}\right) ;{ }^{1} \mathrm{H} \mathrm{NMR}\left(400 \mathrm{MHz}, \mathrm{CDCl}_{3}\right): \delta 6.85,6.69$ (atropoisomers, d, $J=3.2$ $\mathrm{Hz}, \mathrm{d}, J=2.8 \mathrm{~Hz}, 1 \mathrm{H}$ ), 6.68, 6.60 (atropoisomers, d, $J=2.8 \mathrm{~Hz}$, d, $J=3.2 \mathrm{~Hz}, 1 \mathrm{H}$ ), 6.16-6.29 (complex series of m, 3H), 5.87-5.98 (complex series of m, 2H), 5.58 (ddd app. quint., $J=7.2$, 
$14.8 \mathrm{~Hz}, 1 \mathrm{H}$ ), 5.42 (ddd, $J=8.0,14.8,16.2 \mathrm{~Hz}, 1 \mathrm{H}), 5.15-5.22(\mathrm{~m}, 2 \mathrm{H}), 5.09$ (t, $J=9.6 \mathrm{~Hz}, 1 \mathrm{H})$, 4.99 (br. d, $J=18.0 \mathrm{~Hz}, 1 \mathrm{H}$ ), 4.90 (br. d, $J=13.3 \mathrm{~Hz}, 1 \mathrm{H}$ ), 4.79-4.84 (complex series of m, 1H), 4.41 (A of ABq, $J=11.2 \mathrm{~Hz}, 1 \mathrm{H}), 4.30$ (m, 1H), 4.26 (B of ABq, $J=11.2 \mathrm{~Hz}, 1 \mathrm{H}), 4.10-4.22$ (m, 1H), 3.73, 3.72 (atropoisomers, s, s, 3H), 3.62, 3.55 (atropoisomers, s, s, 3H), 3.43-3.49 (m, 1H), 3.23, 3.20 (atropoisomers, s, s, 3H), 2.59-2.65 (m, 2H), 2.54, 2.50 (atropoisomers, t, $J=8.0$ Hz, t, $J=9.2 \mathrm{~Hz}, 1 \mathrm{H}$ ), 2.38 (A of ABq, $J=6.0,15.4 \mathrm{~Hz}, 1 \mathrm{H}$ ), 2.29-2.35 (obsc. m, 1H), 2.29 (B of ABq, $J=4.4,15.4 \mathrm{~Hz}, 1 \mathrm{H}), 2.08-2.24$ (M, 1H), 1.84-1.94 (m, 2H), 1.72, 1.70 (atropoisomers, s, s, 3H), 1.04 (br. s, 24 H), 0.84 (s, 9H), -0.01, -0.02 (atropoisomers, s, s, 3H), -0.06 (s, 3H); ${ }^{13} \mathrm{C}$ NMR (75 MHz, $\left.\mathrm{CDCl}_{3}\right): \delta 173.0,172.4,156.1,156.0,147.6,137.5,137.4,136.1,135.1,135.0$, 133.8, 133.7, 133.3, 133.0, 132.9, 131.5, 126.6, 118.6, 118.3, 116.3, 116.0, 115.4, 112.9, 112.6, 97.1, 82.4, 81.8, 79.2, 78.6, 78.5, 78.1, 72.2, 71.5, 61.4, 56.7, 56.6, 55.6, 42.0, 40.5, 40.2, 39.8, 29.7, 28.7, 26.1, 18.4, 18.3, 13.0, 9.9, -3.0, -4.1; IR (neat) $v_{\max } 2930,2857,1699,1589,1428$, 1215, 1112, 1053, 745; CIMS ( $\mathrm{NH}_{3}$ gas): 945.4, 913.2, 888.6, 706.3, 674.2, 556.4, 490.1, 458.1, 352.1, 317.2, 204.1, 97.1; HRMS (CI, $\mathrm{NH}_{3}$ ) m/z calcd for $\mathrm{C}_{48} \mathrm{H}_{80} \mathrm{Cl}_{3} \mathrm{NO}_{7} \mathrm{Si}_{2}[\mathrm{M}]^{+}$943.454, found: 943.452.

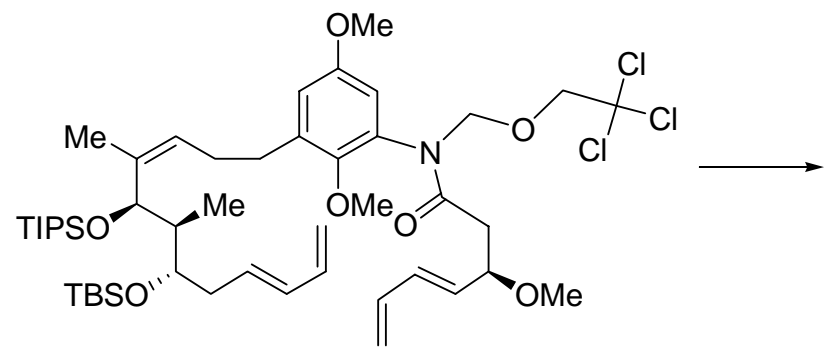

28

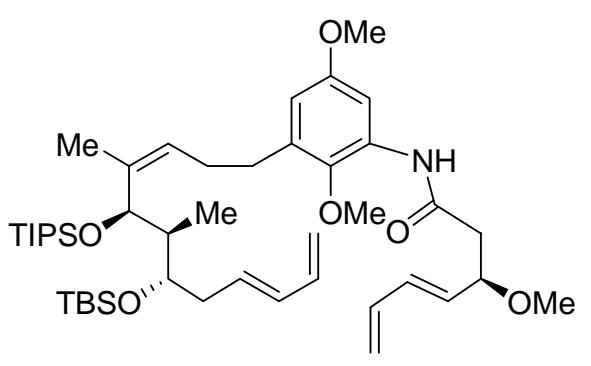

6

\section{[1(3Z,5R,6S,7S,9E),3R,4E]-3-Methoxy-hepta-4,6-dienoic $\quad$ acid $\quad$ [3-[7-(tert-butyl-} dimethylsilyloxy)-4,6-dimethyl-5-triisopropylsilyloxy-dodeca-3,9,11-trienyl]-2,5-dimethoxyphenyl\}-amide 6. To a solution of 28 (118 mg, $0.125 \mathrm{mmol})$ in $\mathrm{MeOH}(16 \mathrm{~mL})$ was added anhydrous sodium hydrogenphosphate $(72 \mathrm{mg}, 0.514 \mathrm{mmol})$ at $-30{ }^{\circ} \mathrm{C}$. The resulting mixture was stirred at this temperature for 10 minutes, treated with sodium mercury amalgam (5\% Na, $1.95 \mathrm{~g}$ ) and kept under vigorous stirring between $-20^{\circ} \mathrm{C}$ and $-30{ }^{\circ} \mathrm{C}$ for 80 minutes. The crude reaction mixture was filtrated over a short plug of silica gel (rinsed several times with AcOEt), 
concentrated and dissolved in AcOEt and water. The organic layer was separated and the aqueous layer was extracted with AcOEt. The combined organic extracts were washed with brine, dried over $\mathrm{MgSO}_{4}$, filtered and concentrated. The crude residue was purified by flash chromatography over silica gel (hexanes/AcOEt: 9/1) to give 6 as a colorless oil (74 mg, 0.094 mmol, 75 \%). $[\alpha]_{\mathrm{D}}^{20}+14.7$ (c 1.03, $\left.\mathrm{CHCl}_{3}\right) ;{ }^{1} \mathrm{H}$ NMR (400 MHz, $\left.\mathrm{CDCl}_{3}\right): \delta 8.96(\mathrm{~s}, 1 \mathrm{H}), 7.91$ (d, $J=2,8 \mathrm{~Hz}, 1 \mathrm{H}$ ), 6.40 (d, $J=3.2 \mathrm{~Hz}, 1 \mathrm{H}), 6.16-6.37$ (complex series of m, 3H), 5.91 (dd, $J=$ 10.8, 14.8 Hz, 1H), 5.58 (dd, $J=8.0,14.0 \mathrm{~Hz}, 2 \mathrm{H}$ ), 5.14-5.27 (complex series of m, 2H), 5.23 (obsc. t, $J=6.0 \mathrm{~Hz}, 1 \mathrm{H}$ ), 4.96 (app. d, $J=16.8 \mathrm{~Hz}, 1 \mathrm{H}$ ), 4.89 (app. d, $J=10.4 \mathrm{~Hz}, 1 \mathrm{H}$ ), 4.30 (d, $J$ $=9.6 \mathrm{~Hz}, 1 \mathrm{H}), 4.07$ (td, $J=4.0,7.8 \mathrm{~Hz}, 1 \mathrm{H}), 3.76$ (s, 3H), 3.66 (s, 3H), 3.45 (br. d, $J=10.0 \mathrm{~Hz}$, $1 \mathrm{H}), 3.37$ (s, 3H), 2.58-2.69 (complex series of m, 4H), 2.35-2.45 (m, 1H), 2.16-2.22 (m, 1H), 2.06-2.13 (m, 1H), 1.88 (br. q, J = 6.8 Hz, 2H), 1.71 (s, 3H), 1.04, 1.03 (2s, 21H), 1.02 (d, $J=6.9$ $\mathrm{Hz}, 3 \mathrm{H}), 0.84$ (s, 9H), -0.02 (s, 3H), -0.07 (s, 3H); ${ }^{13} \mathrm{C}$ NMR (75 MHz, $\mathrm{CDCl}_{3}$ ): $\delta$ 169.2, 156.3, 141.1, 137.3, 137.1, 135.9, 134.9, 134.7, 133.4, 133.0, 132.6, 131.5, 126.9, 119.1, 115.1, 110.4, 103.7, 78.8, 72.5, 72.2, 61.2, 56.5, 55.6, 44.5, 44.2, 35.4, 30.0, 29.3, 25.9, 18.3, 18.2, 18.0, 12.7, 10.6, -4.4; IR (neat) $v_{\max }$ 3305, 2928, 2864, 1689, 1530, 1463, $1056 \mathrm{~cm}^{-1}$; CIMS (NH $\mathrm{NH}_{3}$ gas: 783.9, 740.9, 726.8, 636.4, 478.3, 399.3, 330.1, 218.0, 192.1, 115.1, 97.1; HRMS (CI, $\left.\mathrm{NH}_{3}\right) \mathrm{m} / \mathrm{z}$ calcd for $\mathrm{C}_{45} \mathrm{H}_{77} \mathrm{NO}_{6} \mathrm{Si}_{2}[\mathrm{M}]^{+}$783.529, found: 783.523 .

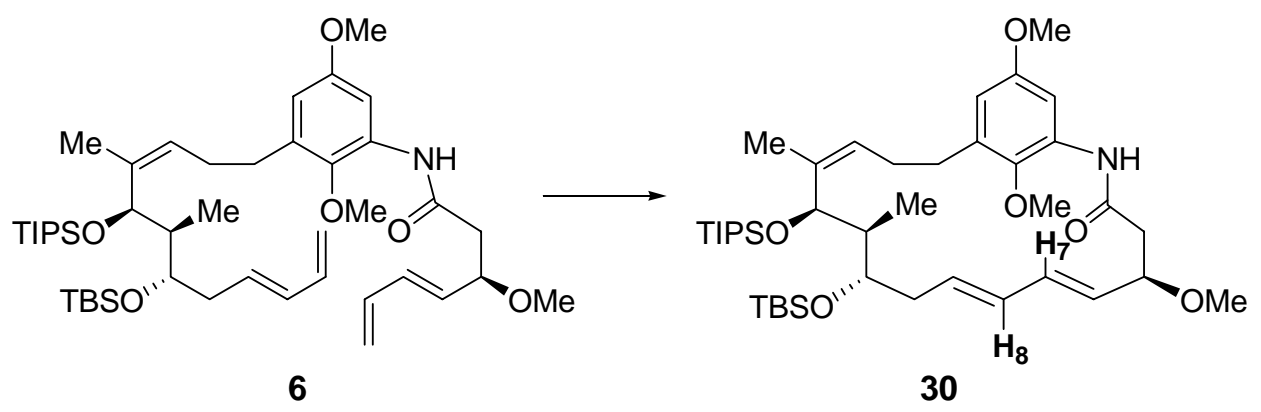

(5R,6E,8E,11S,12S,13R,14Z)-11-(tert-butyl-dimethylsilyloxy)-5,20,22-trimethoxy-12,14dimethyl-13-triisopropylsilyloxy-2-aza-bicyclo[16.3.1]docosa-1(22),6,8,14,18,20-hexaen-3one 30. In a $15 \mathrm{~mL}$ pressure tube was dissolved bis-diene 6 (5.0 mg, $6.4 \mu \mathrm{mol})$ in degassed dichloromethane (3 mL, $0.002 \mathrm{M}$ ) and tricyclohexylphosphine [1,3-bis(2,4,6-trimethylphenyl)4,5-dihydroimidazol-2-ylidene][benzylidine] ruthenium (IV) dichloride 29 (0.5 mg, $0.6 \mu$ mol, 
10 mol\%) was added. The resulting solution was heated to reflux for 2 hours, concentrated and purified by flash chromatography over silica gel (gradient from hexanes/AcOEt: 98/2 to 90/10) to afford the cyclic diene $(1.9 \mathrm{mg}, 2.6 \mu \mathrm{mol}, 41 \%)$ as a pale oil. $[\alpha]_{\mathrm{D}}^{20}+4.2\left(c 0.27, \mathrm{CHCl}_{3}\right) ;{ }^{1} \mathrm{H}$ NMR (400 MHz, $\mathrm{CDCl}_{3}$ ): $\delta 8.73$ (br. s, 1H), 7.75 (d, $\left.J=2.9 \mathrm{~Hz}, 1 \mathrm{H}\right), 6.54$ (dd, $J=10.6,15.6$ Hz, 1H, H7), 6.35 (d, $J=3.2$ Hz, 1H), 5.88 (dd, $J=10.6,16.1 \mathrm{~Hz}, 1 \mathrm{H}, \mathbf{H}_{8}$ ), 5.50-5.61 (m, 1H), 5.30 (dd, $J=5.4,15.6 \mathrm{~Hz}, 1 \mathrm{H}), 5.24$ (m, 1H), 4.11-4.15 (m, 1H), 3.74-3.77 (obsc. m, 1H), 3.74 (s, 3H), 3.69 (s, 3H), 3.48-3.52 (obsc. m, 1H), 3.48 (s, 3H), 2.98 (A of ABX, $J=4.4,15.8 \mathrm{~Hz}$, 1H), 2.72-2.80 (m, 2H), 2.52 (B of ABX, $J=3.6,15.8 \mathrm{~Hz}, 1 \mathrm{H}), 2.02-2.08$ (m, 3H), 1.80-1.85 (m, 2H), 1.72 (s, 3H), 1.02 (s, 21H), 0.93 (d, $J=6.8 \mathrm{~Hz}, 3 \mathrm{H}), 0.82$ (s, 9H), -0.01 (s, 3H), -0.02 (s, 3H); ${ }^{13} \mathrm{C}$ NMR (75 MHz, $\mathrm{CDCl}_{3}$ ): $\delta$ 174.8, 155.2, 147.8, 145.1, 137.5, 137.3, 128.2, 126.9, 124.6, 120.3, 112.1, 110.7, 103.7, 78.5, 71.8, 65.4, 60.7, 58.7, 57.3, 43.2, 39.0, 36.3, 30.1, 29.4, 26.6, 18.5, 18.3, 12.6, 10.2, -4.4, -4.8; CIMS ( $\mathrm{NH}_{3}$ gas): 729.5, 697.5, 636.4, 397.4, 217.2, 97.1; HRMS (CI, $\mathrm{NH}_{3}$ ) m/z calcd for $\mathrm{C}_{41} \mathrm{H}_{72} \mathrm{NO}_{6} \mathrm{Si}_{2}[\mathrm{M}+\mathrm{H}]^{+}$730.490, found: 730.486 .

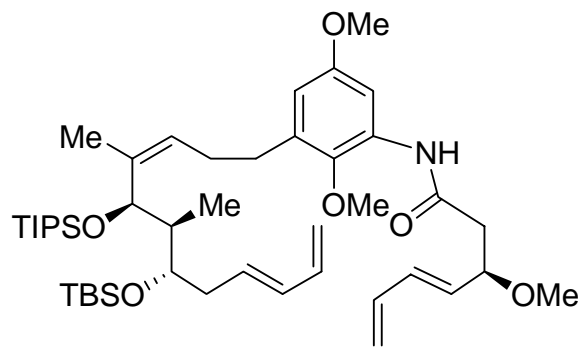

6

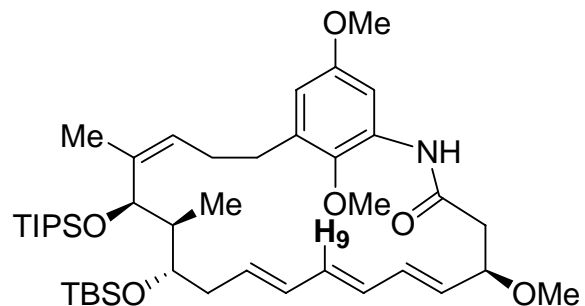

5

(5R,6E,8E,10E,13S,14S,15R,16Z)-13-(tert-butyl-dimethylsilyloxy)-5,22,24-trimethoxy-14,16dimethyl-15-triisopropylsilyloxy-2-aza-bicyclo[18.3.1]tetracosa-1(24),6,8,10,16,20,22-

heptaen-3-one 5. To a solution of bis-diene 6 (68 mg, $87 \mu \mathrm{mol})$ in dichloromethane (43 mL, $0.002 \mathrm{M}$ ) was added benzylidene-bis(tricyclohexylphosphine)dichlororuthenium 31 (7.1 mg, 8.6 $\mu \mathrm{mol}, 10 \mathrm{~mol} \%)$. The resulting orange-purple solution was heated to reflux for 15 hours, another $10 \mathrm{~mol} \%$ of Grubbs catalyst $(7.1 \mathrm{mg}, 8.6 \mu \mathrm{mol})$ were added and the reaction was heated to reflux for another 5 hours. The reaction mixture was then cooled to rt and air was passed through the solution for 15 minutes at which time the crude mixture was concentrated and purified by flash chromatography over silica gel (gradient from hexanes/AcOEt: 9/1 to 7/3) to afford $8.2 \mathrm{mg}$ 
of the starting material 6 and the desired cyclic triene 5 (48 mg, $63.5 \mu \mathrm{mol}, 73$ \%, 83 \% based on recovered starting material) as a pale yellow foam. $[\alpha]_{\mathrm{D}}^{20}+13.5\left(c 0.72, \mathrm{CHCl}_{3}\right) ;{ }^{1} \mathrm{H} \mathrm{NMR}(400$ $\mathrm{MHz}, \mathrm{CDCl}_{3}$ ): $\delta 7.88$ (d, $\left.J=2,8 \mathrm{~Hz}, 1 \mathrm{H}\right), 7.83$ (s, 1H), 6.30 (d, $\left.J=3.2 \mathrm{~Hz}, 1 \mathrm{H}\right), 6.17$ (dd, $J=$ 8.0, 15.6 Hz, 1H, $\mathbf{H}_{9}$ ), 5.84-5.88 (complex series of m, 3H), 5.53 (dd, $J=6.8,15.4 \mathrm{~Hz}, 1 \mathrm{H}$ ), 5.42 (dt, $J=6.5,15.4 \mathrm{~Hz}, 1 \mathrm{H}), 5.25$ (t, $J=6.0 \mathrm{~Hz}, 1 \mathrm{H}), 4.21$ (d, $J=10.8 \mathrm{~Hz}, 1 \mathrm{H}), 4.05-4.14$ (m, 2H), 3.75 (s, 3H), 3.67 (s, 3H), 3.51-3.54 (m, 1H), 3.37 (s, 3H), 2.80 (A of ABX, $J=4.0,12.8 \mathrm{~Hz}$, 1H), 2.74 (B of ABX, J = 4.2, $12.8 \mathrm{~Hz}, 1 \mathrm{H}$ ), 2.50 (dd, $J=12.8,9.2 \mathrm{~Hz}, 1 \mathrm{H}$ ), 2.34 (td, $J=12.8$, $5.2 \mathrm{~Hz}, 1 \mathrm{H}), 2.11-2.15$ (m, 2H), 2.02-2.9 (m, 1H), 1.88 (br. q, J = 6.8 Hz, 1H), 1.74 (s, 3H), 1.001.06 (m, 21H), 0.99 (d, $J=7.2 \mathrm{~Hz}, 3 \mathrm{H}), 0.83$ (s, 9H), 0.01 (s, 3H), -0.01 (s, 3H); ${ }^{13} \mathrm{C}$ NMR (75 $\left.\mathrm{MHz}, \mathrm{CDCl}_{3}\right): \delta 173.8,156.0,147.8,147.1,138.1,137.8,137.7,134.6,126.9,119.1,115.9$, 115.8, 113.8, 113.7, 113.0, 82.3, 70.0, 62.2, 61.1, 60.0, 55.5, 42.0, 38.9, 36.3, 30.1, 29.1, 25.6, $18.4,18.3,12.7,10.3,-4.0,-5.4$; IR (neat) $v_{\max } 3305,2924,2847,1692,1461 \mathrm{~cm}^{-1}$; CIMS $\left(\mathrm{NH}_{3}\right.$ gas): 756.5, 738.6, 636.4, 472.5, 302.1, 192.1, 97.1; HRMS (CI, $\mathrm{NH}_{3}$ ) m/z calcd for $\mathrm{C}_{43} \mathrm{H}_{74} \mathrm{NO}_{6} \mathrm{Si}_{2}[\mathrm{M}+\mathrm{H}]^{+}$756.505, found: 756.509 . 